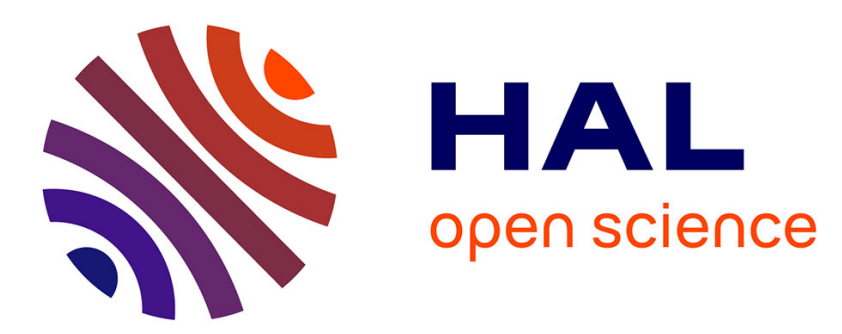

\title{
Fluctuating ionic polarizabilities in the condensed phase: first-principles calculations of the Raman spectra of ionic melts
}

\author{
Paul A Madden, Robert James Heaton
}

\section{- To cite this version:}

Paul A Madden, Robert James Heaton. Fluctuating ionic polarizabilities in the condensed phase: first-principles calculations of the Raman spectra of ionic melts. Molecular Physics, 2008, 106 (12-13), pp.1703-1719. 10.1080/00268970802029497 . hal-00513188

\author{
HAL Id: hal-00513188 \\ https://hal.science/hal-00513188
}

Submitted on 1 Sep 2010

HAL is a multi-disciplinary open access archive for the deposit and dissemination of scientific research documents, whether they are published or not. The documents may come from teaching and research institutions in France or abroad, or from public or private research centers.
L'archive ouverte pluridisciplinaire HAL, est destinée au dépôt et à la diffusion de documents scientifiques de niveau recherche, publiés ou non, émanant des établissements d'enseignement et de recherche français ou étrangers, des laboratoires publics ou privés. 


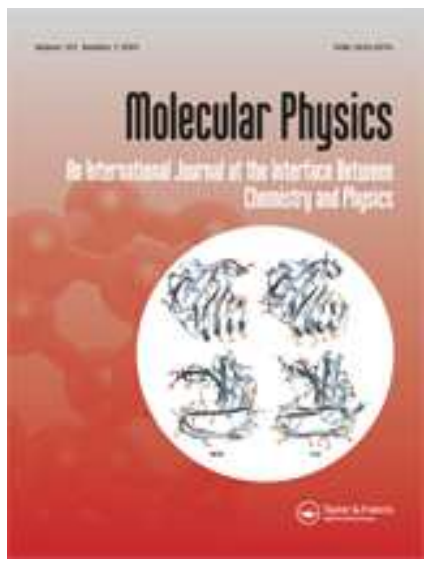

Fluctuating ionic polarizabilities in the condensed phase: first-principles calculations of the Raman spectra of ionic melts

\begin{tabular}{|c|c|}
\hline Journal: & Molecular Physics \\
\hline Manuscript ID: & TMPH-2008-0034.R1 \\
\hline Manuscript Type: & Full Paper \\
\hline $\begin{array}{r}\text { Date Submitted by the } \\
\text { Author: }\end{array}$ & 20-Feb-2008 \\
\hline Complete List of Authors: & $\begin{array}{l}\text { Madden, Paul; Edinburgh University, School of Chemistry } \\
\text { Heaton, Robert; University of Edinburgh, Chemistry }\end{array}$ \\
\hline Keywords: & $\begin{array}{l}\text { Raman Spectroscopy, Ionic Liquids, Polarizability, Computer } \\
\text { Simulation, LiF and BeF2 }\end{array}$ \\
\hline \multicolumn{2}{|c|}{$\begin{array}{l}\text { Note: The following files were submitted by the author for peer review, but cannot be converted } \\
\text { to PDF. You must view these files (e.g. movies) online. }\end{array}$} \\
\hline Paper.tex & \\
\hline
\end{tabular}

\section{S) ScholaroNE" \\ Manuscript Central}




\title{
Fluctuating ionic polarizabilities in the condensed phase: first-principles calculations of the Raman spectra of ionic melts
}

\author{
Robert J. Heaton and Paul A. Madden \\ School of Chemistry, University of Edinburgh, Edinburgh EH9 3JJ, UK
}

(Dated: February 20, 2008)

\begin{abstract}
An approach to calculating Raman spectra of ionic materials from first principles is described; the method is applicable to molten systems which cannot be treated by summing the contributions from normal modes of vibration. The approach offers a way to validate a simulation by comparison with a Raman spectrum; for many materials under extreme conditions of temperature or pressure, Raman spectroscopy may be the only practicable experimental window on the structure at the atomic scale. The method involves the direct calculation of the time correlation functions of the polarizability fluctuations in the sample, which involves the introduction of a model for the dependence of the polarizability on the ionic coordinates. The model is parameterized by fitting a large number of values of the polarizabilities of individual ions in condensed phase environments. These are calculated, using a recently introduced $a b$ initio method, from the response of the electron density to an applied electric field on a series of configurations obtained from molecular dynamics simulations of the material of interest. The results of the calculations are compared with experimental spectra on liquid and solid $\mathrm{LiF}$, liquid $\mathrm{BeF}_{2}$, and $\mathrm{LiF}: \mathrm{BeF}_{2}$ mixtures. The spectra are well reproduced by the method, particularly the isotropic components which are generally most useful for the diagnosing coordination structures in experimental studies. Since the $a b$ initio methodology has already been shown to work for oxides, the method should be applicable melts of geophysical interest inter alia.
\end{abstract}




\section{INTRODUCTION}

There is considerable interest in developing the capability to calculate Raman spectra of disordered ionic materials from first principles. From a theoretical perspective, ionic materials are strongly interacting closed-shell systems, and so push to the limit a description of their properties through the theory of intermolecular forces ${ }^{1}$. In materials with polyvalent cations, the spectra contain distinctive bands which are the signatures of particular coordination structures ${ }^{2}$. Raman spectra may be especially sensitive to particular intermediate-range structures, as with the signature of boroxyl rings in the spectra of borate glasses ${ }^{3,4}$. In the molten state, the Raman spectrum may be the only experimental technique capable of giving this structural information because the corrosive nature of the high temperature melts prevents the design of suitable containers or because the melt of interest contains so many components that the decomposition of a diffraction pattern into partial structure factors is impracticable. Raman spectra of molten fluorides have been obtained by using windowless cells ${ }^{5}$ and the use of levitated droplets has enabled the spectra of molten oxides to be obtained ${ }^{6}$. This type of information is valuable to understand the properties of the melts involved in electrofining ${ }^{5}$ or in various geophysical processes ${ }^{7,8}$. Nevertheless, the conversion of the pattern of bands seen in a spectrum into structural information is not straightforward ${ }^{9}$. In dilute solutions of a polyvalent cation in a simple salt, the spectra of discrete molecular ions may be recognised by their polarization characteristics and the pattern of band frequencies ${ }^{2}$, but in more concentrated melts, these coordination polyhedra overlap, networks form and the direct interpretation of the spectrum becomes more hazardous. Progress could be made by calculation of a Raman spectrum in a computer simulation: if the calculated spectrum agrees sufficiently well with the experimental one to validate the representation of the real material by the simulation, the detailed information it generates may be interrogated to provide the desired level of structural information or to predict the values of quantities which cannot be measured directly.

The general expression for the light-scattering spectrum of a sample at frequency shift $\omega$ is ${ }^{10}$

$$
I_{a b}(\mathbf{q}, \omega) \propto\left|E_{b}^{0}\right|^{2} \Re \int_{0}^{\infty} d t e^{i \omega t}\left\langle\Pi_{a b}(\mathbf{q}, t) \Pi_{a b}(\mathbf{q}, 0)\right\rangle
$$

where $a$ and $b$ are the directions of polarization of the incident $\left(\mathbf{E}^{0}\right)$ and scattered electric fields and $\mathbf{q}$ is the scattering vector, determined by the wavelength of the radiation and the scattering angle. $\Pi_{a b}(\mathbf{q}, t)$ is a spatial fourier component of the instantaneous value of the $a b^{\text {th }}$ component of the polarizability tensor of the sample. Raman scattering is caused by the fluctuations in the polarizability which are caused by interactions between atoms at relatively short interparticle separations compared to the wavelength of light so the spatial correlation between the fluctuations extends only to small distances and we can safely replace equation 1 by its $q \rightarrow 0$ limit and calculate the correlation function of the fluctuating part of the polarizability 
$\Delta \Pi_{a b}(t)=\Pi_{a b}(t)-\left\langle\Pi_{a b}\right\rangle$ in what follows. If the material is such that the atoms are making small amplitude vibrations about some equilibrium position then the Raman spectrum may be calculated by expanding the polarizability about its value in the equilibrium configuration in terms of the normal modes of vibration, $\left\{Q_{k}\right\}$, with harmonic frequencies $\left\{\omega_{k}\right\}$,

$$
\Pi_{\alpha \beta}=\left(\Pi_{\alpha \beta}\right)_{0}+\sum_{k}\left[\left(\frac{\partial \Pi_{\alpha \beta}}{\partial Q_{k}}\right)_{0} Q_{k}\right]+\text { higher-order terms. }
$$

Substituting this expression into equation 1, retaining only the first derivatives, leads to the usual expression for the Raman spectrum in terms of normal mode frequencies

$$
I_{a b}(\omega) \propto\left|E_{b}^{0}\right|^{2} \sum_{k}\left(\frac{\partial \Pi_{a b}}{\partial Q_{k}}\right)_{0}^{2} \frac{k_{B} T}{\hbar \omega_{k}} \delta\left(\omega-\omega_{k}\right) .
$$

Recently, several groups of workers ${ }^{4,11,12}$ have shown how the Raman spectra of glasses may be calculated from first principles via equation 3. The normal modes and polarizability derivatives, $\left(\partial \Pi / \partial Q_{k}\right)_{0}$, are evaluated from density functional perturbation theory at a series of glass configurations generated in $a b$ initio molecular dynamics simulations and the Raman spectrum calculated as an average over the spectra obtained from these configurations. Using these methods, Pasquarello and co-workers ${ }^{4,13}$ have cast light on a number of long-standing controversies in the spectroscopy of glasses, such as the contribution of boroxyl ring vibrations to the spectra of $\mathrm{B}_{2} \mathrm{O}_{3}$. However, these methods cannot readily be applied to liquids and melts as the step of finding normal modes of vibration about local equilibrium geometries is not directly applicable. It might, in principle, be possible to make progress using "instantaneous normal modes"14 or the normal modes of the significant structures ${ }^{15}$. However, many of these modes occur at low, or imaginary frequencies and are highly anharmonic, which will jeopardize the truncation of the expansion at the linear term in equation 2. An alternative way of approaching the calculation of the Raman spectrum, which avoids these limitations, is to obtain an expression for the dependence of the polarizability on the positions of all the atoms and then compute the correlation functions of this expression from an MD trajectory. Previous attempts to follow this route have been limited by the difficulty of constructing a suitable model for the polarizability ${ }^{8,23}$.

The starting point for obtaining such an expression is to relate the polarization of the sample in the presence of the external field $\mathbf{E}^{0}$,

$$
\mathbf{P}=\boldsymbol{\Pi} \cdot \mathbf{E}^{0}
$$

to the field- induced dipole moments of the individual atoms $\left\{\mathbf{p}^{i}\right\}$ :

$$
\mathbf{P}(\mathbf{r})=\sum_{i}^{N} \mathbf{p}^{i} \delta\left(\mathbf{r}-\mathbf{r}^{i}\right)
$$


Provided that the field does not induce charge-transfer between different atoms (i.e. for normal dielectric media) $)^{16-18}$

$$
\mathbf{p}^{i}\left(\left\{\mathbf{r}^{N}\right\}\right)=\boldsymbol{\alpha}^{i}\left(\left\{\mathbf{r}^{N}\right\}\right) .\left(\mathbf{E}^{0}\left(\mathbf{r}^{i}\right)-\sum_{j \neq i} \mathbf{T}^{i j} \cdot \mathbf{p}^{j}\left(\left\{\mathbf{r}^{N}\right\}\right)\right) .
$$

The term in brackets on the right-hand side of this expression is the instantaneous value of the local field at ion $i$, it consists of the external field, $\mathbf{E}^{0}$, plus the re-radiated fields from the dipoles induced in all the other ions of the sample, $\mathbf{T}^{i j}$ being the dipole-dipole interaction tensor between atoms $i$ and $j\left(T_{\alpha \beta}^{i j}=\right.$ $\left.\nabla_{\alpha} \nabla_{\beta}\left(r^{i j}\right)^{-1}\right)$. The first term is the instantaneous dipole polarizability of ion $i, \boldsymbol{\alpha}^{i}\left(\left\{\mathbf{r}^{N}\right\}\right)$, it depends on the positions of all other ions in the sample, since interactions with them change the value of the polarizability. The individual dipole of ion $i$ therefore acquires a time modulation due to its dependence on the relative positions of the other ions $j$ in the sample, through $\boldsymbol{\alpha}^{i}\left(\left\{\mathbf{r}^{N}\right\}\right)$ and through the dipole-induced dipole terms involving $\mathbf{T}^{i j}$. It is these fluctuations which are responsible for the Raman spectrum.

In the past, we have developed an expression for $\boldsymbol{\alpha}^{i}\left(\left\{\mathbf{r}^{N}\right\}\right)$ for an ionic material ${ }^{19-21}$, and obtained values for the parameters it contains by combining various scaling arguments with a limited set of quantum chemical calculations of the polarizabilities of small clusters ${ }^{22}$. By these means we have been able to reproduce Raman spectra of molten salts, including strongly interacting systems like trivalent metal halides $^{9,23,24}$. These have allowed us to comment on the assignment of spectral features to particular structural units present in the melts. The calculations have been restricted to a semi-quantitative comparison with experiment due to our inability to really pin down the polarizability model because of the problem of decomposing the $a b$ initio calculated polarizability of a cluster into a set of contributions attributable to the individual atoms due to basis set superposition errors, dipole-induced dipole terms etc. ${ }^{25}$.

Recently we introduced a new development in the first-principles calculation of condensed phase polarizabilities ${ }^{26}$ which suggests a way of overcoming this bottleneck. The individual ionic polarizabilities appropriate to particular condensed ionic configurations are calculated directly from a first-principles calculation on a periodically replicated supercell. In reference ${ }^{26}$ the mean polarizabilities of oxide ions (as well as several cations) in a variety of ionic materials were calculated and compared with experimental values (obtained from refractive indices via the Lorentz-Lorenz formula) and with previous calculations ${ }^{27}$. Despite the fact that the polarizabilities of oxide ions vary enormously from one material to another the calculations reproduced the accepted values extremely well. Subsequently, the method has been tested on condensed fluorides, with equally good agreement with experiment ${ }^{28}$. Since this method can be applied to arbitrary condensed phase configurations, such as those obtained from MD runs on a melt, we can use it to generate many $\boldsymbol{\alpha}^{i}\left(\left\{\mathbf{r}^{N}\right\}\right)$ values for representative ionic arrangements for the melt. We can then use this large dataset to optimize the parameters in a general expression for $\boldsymbol{\alpha}^{i}\left(\left\{\mathbf{r}^{N}\right\}\right)$, for example, by minimizing 
the difference between the $a b$ initio-calculated values and the values obtained from the expression when evaluated with exactly the same ionic configurations as used to generate the $a b$ initio data.

We have made extensive use of a similar "force-fitting" idea ${ }^{29}$ to obtain $a b$ initio parameterized polarizable force-fields for ionic materials ${ }^{30,31}$. The quantitative success ${ }^{32}$ of this procedure, in generating force-fields which predict observables without loss of ab initio accuracy, has encouraged this extension to the Raman scattering. Of course, the quality of the spectra will be affected by the reliability of the model expression for $\boldsymbol{\alpha}^{i}\left(\left\{\mathbf{r}^{N}\right\}\right)$. Here we will make use of the expression suggested in the earlier work ${ }^{20}$, but alternative expressions based upon different ideas about the representation of the interaction-induced contributions to the polarizability could be investigated in future work; for example, a bond-polarizability model could be tried.

In the present paper we will describe spectra calculated from equation 1 with an $a b$ initio parametrized model for the polarizability. The time correlation functions will be evaluated from molecular dynamics simulations generated with $a b$ initio optimized polarizable interaction potentials obtained by force-fitting. The optimization of the polarizability model and the interaction potential are performed on samples containing $\sim 100$ ions, for which the $a b$ initio calculations are viable, but the subsequent MD calculations to obtain the time correlation functions are performed on considerably larger samples, to ensure that the interparticle interactions are sampled correctly. The systems on which we will evaluate the procedure are $\mathrm{LiF}, \mathrm{BeF}_{2}$ and their liquid mixtures. $\mathrm{BeF}_{2}$ is a structural analogue of $\mathrm{SiO}_{2}{ }^{33}$ and readily forms a tetrahedrally coordinated network glass. Galeener $e t a l^{34}$ noted strong similarities between the Raman spectra of $\mathrm{BeF}_{2}, \mathrm{SiO}_{2}$ and $\mathrm{GeO}_{2}$, which reflect their common network structure. Mixing alkali fluorides with $\mathrm{BeF}_{2}$ breaks down the network to form mixtures with similar properties to alkali silicates; this process results in major changes in the Raman spectra and one challenge for simulation is to relate this evolution in the spectrum to structural features. The $\mathrm{LiF}: \mathrm{BeF}_{2}$ mixture becomes the material of choice as a solvent for actinide ions in the moltensalt reactor ${ }^{35}$ and as a heat-exchanger or blanket material in proposed breeder and fusion reactors ${ }^{36}$. Because of these potential applications, an enormous amount of experimental work has been done on $\mathrm{LiF}^{-\mathrm{BeF}_{2}}{ }^{37}$, including Raman studies ${ }^{38}$. Alongside cryolite ${ }^{5,24}$, the electrolyte involved in aluminium extraction, it is by far the best-characterized fluoride melt. We have already determined interaction potentials for these mixtures and compared simulation results with a wide range of observable properties ${ }^{32,39,40}$ in the liquid and solid state.

Besides examining the Raman spectra of the fluids, we will begin by presenting results for solid $\mathrm{LiF}$. LiF crystallizes in the rocksalt structure whose high symmetry means that there are no Raman-active lattice vibrations ${ }^{41}$, i.e. no Raman spectrum is predicted by using equation 2 truncated at first order (as in all direct methods for calculating a Raman spectrum from first principles). The observed spectrum reflects the 
two-phonon density of states (DoS) and, as such, exhibits a number of sharp features arising from critical points in the $\operatorname{DoS}^{41}$. The ability to reproduce these spectra is a very exacting test of both the polarizability model and the interaction potential. Furthermore, we previously parametrized a polarizability model for solid LiF by adapting quantum chemical methods ${ }^{19,20}$. We can use the parameter values obtained in this work to assess the reliability of the parameters emerging from the fitting methods.

\section{AB INITIO PARAMETRIZATION OF THE FLUCTUATING IONIC POLARIZABILITY MODEL.}

\section{A. Polarizability calculations}

The calculation of the ionic polarizabilities proceeds through three stages. Firstly, we create several sets of ionic configurations for the materials of interest. They are generated by molecular dynamics simulations using high quality interaction potentials obtained by ab initio force-fitting as described previously $31,32,39$. The simulations are performed with periodic boundary conditions on relatively small simulation cells, containing roughly 100 ions, in order to enable us to carry out electronic structure calculations. The simulation conditions are chosen so that configurations selected from them sample as wide a range of coordination environments for the ions as are likely to be sampled in the experimental conditions and typically involve calculations on high temperature crystals as well as the liquid phase. Typically, five ionic configurations might be used as the basis of the parameterization of a model for some material, and given that the polarizability of an ion has six independent components this means that roughly 3000 data values contribute to the refinement of the model.

We then carry out $a b$ initio planewave DFT electronic structure calculations on the selected configurations. We use the CASTEP code ${ }^{42}$ with a planewave cut-off of $1000 \mathrm{eV}$. Only the $\Gamma$-point is included in the Brillouin-zone sampling scheme since our interest is in performing calculations with quite large simulation cells on insulating systems. The calculations are carried out using the $\mathrm{PBE}^{43}$ exchange-correlation functional and norm-conserving pseudopotentials created with this functional and optimized at the cut-off energy using the OPIUM program ${ }^{44}$. As described in detail in reference ${ }^{26}$ we obtain the individual ionic dipoles by transformation of the occupied optimum Kohn-Sham orbitals $\left\{\psi_{\alpha}\right\}_{\alpha=1, M}$ in the presence of an applied electric field to a maximally localised set of Wannier Functions ${ }^{30,45}\left\{u_{\alpha}\right\}_{\alpha=1, M}$. In ionic (closed shell) systems, this transformation localises a set of valence orbitals on each ion and the molecular charge densities obtained from these orbitals has been shown to give excellent values for the ionic multipoles. The electric field-induced dipoles are obtained from the Kohn-Sham orbitals from four electronic structure calculations, consisting of the unperturbed system and the system (at exactly the same atomic positions) in the 


\section{B. The polarizability model}

We will make use of the model for the fluctuating polarizabilities in ionic materials introduced some time ago ${ }^{19-21}$. The idea developed in that model was to use the perfect crystalline environment as a reference point and to consider the effect of distortions in the crystal lattice on the polarizability of an ion. If the distortion occurs some distance away from the ion of interest its effect is to create an electric field (and field gradients) at the site of the ion which modify the ionic polarizability due to hyperpolarization effects, as described by Buckingham ${ }^{1,46}$. Two contributions to these "asymptotic" contributions to the polarizability can be distinguished, which give the effect of a strong local field and a field-gradient respectively:

$$
\begin{aligned}
\alpha_{\alpha \beta}^{i,(\gamma)} & =\sum_{i} \gamma^{i} F_{\alpha}\left(\mathbf{r}^{i}\right) F_{\beta}\left(\mathbf{r}^{i}\right) \\
\alpha_{\alpha \beta}^{i,(B)} & =\sum_{i} B^{i} F_{\alpha \beta}^{\prime}\left(\mathbf{r}^{i}\right) .
\end{aligned}
$$

$\gamma$ and $\beta$ are hyperpolarizabilities of the ion in its reference (crystalline) configuration. The sources of the fields and field gradients are the charges and induced multipoles on the other ions in the sample

$$
F_{\alpha}\left(\mathbf{r}^{i}\right)=\sum_{j \neq i}\left(T_{\alpha}^{i j} q^{j}-T_{\alpha \beta}^{i j} \mu_{\beta}^{j}+\frac{1}{3} T_{\alpha \beta \gamma}^{i j} \theta_{\beta \gamma}^{j}\right)
$$




$$
F_{\alpha \beta}^{\prime}=\frac{\partial F\left(\mathbf{r}^{i}\right)_{\beta}}{\partial r_{\alpha}^{i}}
$$

As such, they are simple functions of the pair separations of the ions and easily incorporated into a computer simulation. $q^{j}, \boldsymbol{\mu}^{j}$, and $\boldsymbol{\theta}^{j}$ are the charge, dipole and quadrupole of ion $j$. The dipoles $\left(\boldsymbol{\mu}^{i}\right)$ included in equation 9 are the dipoles induced by the interionic interactions (as opposed to the $\mathbf{p}^{i}$ induced by the external field) and also occur in the calculations of the interionic forces.

In a high symmetry crystal, where the ion is effectively spherical, $\gamma$ and $B$ are specified by a single number. Previously ${ }^{20,21}$, values for the hyperpolarizabilities were determined using ab initio quantum chemistry calculations ${ }^{47}$ on a small cluster of ions embedded in a point charge lattice. In the present work we will make use of equations (7) and (8) as contributions to $\boldsymbol{\alpha}^{i}\left(\left\{\mathbf{R}^{N}\right\}\right)$ and regard $\gamma$ and $\beta$ as single numbers to be determined from the fits to the $a b$ initio-determined polarizabilities.

Fowler and Madden ${ }^{19}$ recognised a further contribution to the fluctuating polarizability from their distorted crystal calculations. When the distortion of the crystal lattice was in the first neighbour shell around the ion of interest, short-range corrections to the predictions of the asymptotic terms (in equations (7) and (8)) are needed. They were attributed to "dents-in-the-wall" of the Madelung potential at the site of an anion in a high symmetry crystal and short-range overlap interactions with immediate neighbours. They were characterized from the $a b$ initio calculations on distorted embedded clusters. In order to express this effect in a tractable way for a simulation, Madden and Board ${ }^{20,21}$ proposed to represent the short-range contributions with a generalized Drude model

$$
\boldsymbol{\alpha}^{i,(s r)}=\left[\mathbf{k}^{i}\left(\left\{\mathbf{r}^{N}\right\}\right)\right]^{-1}
$$

where $\mathbf{k}^{i}\left(\left\{\mathbf{r}^{N}\right\}\right)$ is a force constant tensor for ion $i$. It was expressed in terms of short-ranged functions of the separation between $i$ and its neigbours in the most general form that can be adopted for a second rank symmetric tensor consistent with a pairwise additive model:

$$
\mathbf{k}^{i}\left(\left\{\mathbf{r}^{N}\right\}\right)=\left(k_{0}+\sum_{j \neq i} A\left(r^{i j}\right)\right) \mathbf{I}+\sum_{j \neq i} B\left(r^{i j}\right)\left(3 \hat{\mathbf{r}}^{i j} \hat{\mathbf{r}}^{i j}-\mathbf{I}\right) .
$$

Here $\mathbf{I}$ is the unit tensor, and $A(r)$ and $B(r)$ are functions describing the effect of the short-range interactions on the isotropic and anisotropic parts of the Drude force constant matrix, and hence the polarizability. They are rapidly decreasing functions of $r . \hat{\mathbf{r}}^{i j}$ is a unit vector in the direction of $\mathbf{r}^{i j}$, and $\hat{\mathbf{r}}^{i j} \hat{\mathbf{r}}^{i j}$ is the outer product of this vector with itself. If all the short-range interactions are removed, the force constant returns to its free value, $k_{0}$, and the free-ion polarizability is then $1 / k_{0}$. The functions chosen for $A$ and $B$ match the exponential which appears in the representation of the short-range repulsion in a Born-Mayer interionic 
potential:

$$
\begin{aligned}
& A\left(r^{i j}\right)=a^{i j} \exp \left[-c^{i j}\left(r^{i j}-\sigma^{i j}\right)\right] \\
& B\left(r^{i j}\right)=b^{i j} \exp \left[-d^{i j}\left(r^{i j}-\sigma^{i j}\right)\right] .
\end{aligned}
$$

In each of these expressions there are only two independent parameters; the $\sigma^{i j}$ are simply the sum of the interionic radii for ions $i$ and $j$, and are introduced in the hope that the other parameters will transfer between different materials.

This representation of the short-range effects was shown to reproduce the limited amount of ab initio information available from the distorted cluster calculations. We propose to use this representation to complete our model for $\boldsymbol{\alpha}^{i}\left(\left\{\mathbf{R}^{N}\right\}\right)$ and determine the parameters $a, b, c, d$ and $k_{0}$ from the fits to the ab initio obtained polarizabilities for a much wider range of distorted geometries using the new method.

As summarized above, the form of the polarizability model was motivated by consideration of the ionic polarizability in high symmetry, high coordination number crystals, where ab initio calculations on representative embedded clusters were possible. For the alkali halides, the resulting spectra ${ }^{20,21}$ were shown to agree well with experimental spectra ${ }^{51}$. However the model has since been used in a more empirical way to represent the fluctuating polarizabilities in melts where this starting point is not obviously appropriate. In cryolite and the trivalent metal halide systems, the coordination number of the anions is only two, which is a marked departure from the original picture. In simulations of these systems ${ }^{9,23,24}$, the representations described above have been used, in order to capture appropriate functional dependence of the polarizability on interionic separations, with the parameters estimated by scaling the values originally obtained in the calculations on alkali halides. A major shortcoming of these simulations has been that without a quantitative understanding of the size of different terms it has not been meaningful to calculate the whole spectrum which, as we will see, is strongly affected by the interference between the different mechanisms. Consequently, we have only been able to calculate "subspectra" associated with each of the mechanisms acting independently. Although, these subspectra have allowed identification of the origin of the distinctive bands seen in the experimental spectra of these polyvalent cation systems, we have not been able to reproduce the distribution of Raman intensities across the whole spectrum. The hope in the present work is that with the newly developed ability to parameterize the whole polarizability model, this limitation will be overcome. 


\section{RESULTS FOR LIF}

\section{A. Fitting the polarizability model}

Within the above model, the polarizability of an ion in an arbitrary condensed phase configuration is given by the sum of equations 7,8 , and 11

$$
\boldsymbol{\alpha}^{i}\left(\left\{\mathbf{R}^{N}\right\}\right)=\boldsymbol{\alpha}^{i,(\gamma)}\left(\left\{\mathbf{R}^{N}\right\}\right)+\boldsymbol{\alpha}^{i,(B)}\left(\left\{\mathbf{R}^{N}\right\}\right)+\boldsymbol{\alpha}^{i,(s r)}\left(\left\{\mathbf{R}^{N}\right\}\right)
$$

The expression contains several parameters, collectively known as $\{\chi\}$, consisting of the hyperpolarizabilities $\gamma, B$, and the parameters of the short-range term $a, b, c, d$ and $k_{0}$. They are to be determined by minimizing the difference between the values predicted by the model with those obtained $a b$ initio for exactly the same ionic configuration.

For each system of interest, the optimum set of parameters is determined by minimizing the objective function

$$
A_{\mathrm{TOT}}(\{\chi\})=\frac{1}{3}\left(A_{\mathrm{ISO}}(\{\chi\})+A_{\mathrm{E}}(\{\chi\})+A_{\mathrm{T}}(\{\chi\})\right),
$$

with respect to variations in $\{\chi\}$, where

$$
\begin{aligned}
A_{\mathrm{ISO}}(\{\chi\}) & =\frac{\sum_{i, C}\left|\alpha_{\mathrm{ISO}}^{i}(C,\{\chi\})-\alpha_{\mathrm{ISO}}^{i}(C, \mathrm{DFT})\right|^{2}}{\sum_{i, C}\left|\alpha_{\mathrm{ISO}}^{i}(C, \mathrm{DFT})\right|^{2}} \\
A_{\mathrm{E}}(\{\chi\}) & =\frac{1}{2} \frac{\sum_{i, a, C}\left|\alpha_{\mathrm{E}, a}^{i}(C,\{\chi\})-\alpha_{\mathrm{E}, a}^{i}(C, \mathrm{DFT})\right|^{2}}{\sum_{i, a, C}\left|\alpha_{\mathrm{E}, a}^{i}(C, \mathrm{DFT})\right|^{2}} \\
A_{\mathrm{T}}(\{\chi\}) & =\frac{1}{3} \frac{\sum_{i, a, C}\left|\alpha_{\mathrm{T}, a}^{i}(C,\{\chi\})-\alpha_{\mathrm{T}, a}^{i}(C, \mathrm{DFT})\right|^{2}}{\sum_{i, a, C}\left|\alpha_{\mathrm{T}, a}^{i}(C, \mathrm{DFT})\right|^{2}}
\end{aligned}
$$

Minimization of the objective function was carried out using the MINUIT package ${ }^{49}$ which allows us to constrain the variations in the parameter values to a pre-set range. In these expressions, the index $i$ runs over atoms, and the sum over $C$ represents the sum over all atomic configurations included in the fit. Rather than sum the differences for each of the six independent elements of the polarizability tensors collectively, we have chosen to use combinations which have a particular symmetry as these will reflect different aspects of the local fluctuations and also stimulate the different polarization mechanisms to different extents. For example, only the short-range and $\gamma$ mechanisms may affect the isotropic polarizability. The first term in equation 16 involves the isotropic part of the polarizability tensor, $\alpha_{\mathrm{ISO}}^{i}=\frac{1}{3}\left(\alpha_{x x}^{i}+\alpha_{y y}^{i}+\alpha_{z z}^{i}\right)$. We have found it convenient to work with the fluctuations of the isotropic terms from their average value, so 


\begin{tabular}{|ccccc|}
\hline system & $A_{\mathrm{TOT}}$ & $A_{\mathrm{ISO}}$ & $A_{\mathrm{E}}$ & $A_{\mathrm{T}}$ \\
\hline LiF crystal & 0.0689 & 0.0317 & 0.1215 & 0.0536 \\
\hline LiF melt & 0.1003 & 0.0491 & 0.1355 & 0.1165 \\
\hline $\mathrm{BeF}_{2}$ melt & 0.0745 & 0.0342 & 0.0869 & 0.1024 \\
\hline${\mathrm{LiF}-\mathrm{BeF}_{2}}$ & 0.1738 & 0.0248 & 0.2344 & 0.2622 \\
\hline
\end{tabular}

TABLE I: The isotropic and anisotropic contributions to chi-squared resulting from fits of the model to the DFT calculated polarizabilities for pure $\mathrm{LiF}$ and $\mathrm{BeF}_{2}$ and for their mixtures.

that prior to the calculation of $A_{\mathrm{ISO}}$ using equation 17 , the average isotropic polarizability taken from the DFT calculations, $\bar{\alpha}_{\mathrm{ISO}}^{\mathrm{DFT}}$, is subtracted from both the model and DFT predicted terms. This ensures that the fluctuating part of the isotropic polarizability dominates the contribution that this term makes to the value of chi-squared. The second and third terms sum the anisotropic contributions of $\mathrm{E}$ and $\mathrm{T}$ symmetries in the octahedral symmetry appropriate to the rocksalt structure of crystalline $\operatorname{LiF},\left\{\alpha_{\mathrm{E}, 1}^{i}=\left(2 \alpha_{z z}^{i}-\alpha_{x x}^{i}-\right.\right.$ $\left.\left.\alpha_{y y}^{i}\right) / 4, \alpha_{\mathrm{E}, 2}^{i}=\left(\alpha_{x x}^{i}-\alpha_{y y}^{i}\right) / \sqrt{ } 12\right\}$, and $\left\{\alpha_{\mathrm{T}, 1}^{i}=\sqrt{ } 3 \alpha_{x y}^{i} / 2, \alpha_{\mathrm{T}, 2}^{i}=\sqrt{ } 3 \alpha_{x z}^{i} / 2, \alpha_{\mathrm{T}, 1}^{i}=\sqrt{ } 3 \alpha_{y z}^{i} / 2\right\}$. In the fluid the $\mathrm{E}$ and $\mathrm{T}$ elements contain equivalent information. Note the factors of $\frac{1}{2}$ and $\frac{1}{3}$ in equations 18 and 19, which ensure an equal weighting, and importance, for each of the different symmetry combinations.

For both the solid and liquid phases of LiF, we took five thermally distorted configurations from a range of high-temperature simulations $(500 \mathrm{~K}-1500 \mathrm{~K})$ using a quadrupole-polarizable interaction potential obtained by $a b$ initio force fitting. This potential has been shown to predict the phonon dispersion curves of crystalline LiF extremely well ${ }^{31}$. Each simulation contained 64 ions. We performed DFT calculations of the ionic polarizabilities as described above ${ }^{26}$. In LiF, only the fluoride ions have significant polarizability fluctuations. A separate fit of the model to the fluoride ion polarizabilities was performed for the solid and liquid phases. The optimum values of the objective function resulting from this procedure are given in table I. For the solid phase, the isotropic polarizabilities are well reproduced, as are the off-diagonal elements (contributions of $\mathrm{T}$ symmetry). Differences in the diagonal elements (contributions of E symmetry) are slightly less well reproduced. For the liquid configurations, the fit is again very good, particularly for the isotropic polarizabilities.

The quality of the fit is illustrated in figures 1 . This figure shows the comparison of the ab initio polarizabilities for each of the 160 fluoride ions (in five configurations each containing 32 fluoride ions) with those predicted by the model with optimized parameters. The black lines join the values from the ab initio calculations, whereas the points indicate the values predicted by the model. We have shown the comparison for the solid phase of LiF; the higher ion numbers are from configurations at higher simulation temperatures, so that the amplitude of the polarizability fluctuations increases towards the right of the figure. Uniformly 

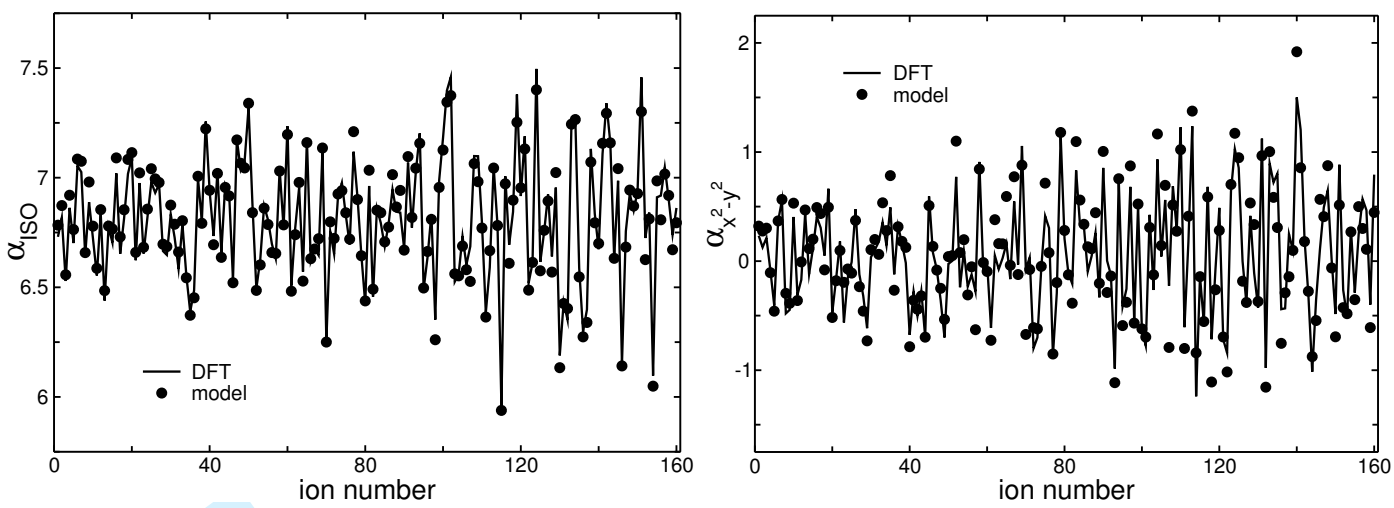

FIG. 1: The figures show values of the isotropic and $x^{2}-y^{2}$ polarizability tensor components for each of $160 \mathrm{~F}^{-}$ions from 5 distorted rocksalt configurations of LiF, each containing $32 \mathrm{~F}^{-}$ions. The solid black line joins the ab initio values whilst the points are the values predicted by the fitted model. The later configurations were taken from higher temperature MD simulations and show greater polarizability fluctuations.

\begin{tabular}{|c|c|c|c|c|}
\hline & LiF solid & $\mathrm{LiF}$ liquid & $\mathrm{BeF}_{2}$ & mixture \\
\hline $\bar{\alpha}^{\mathrm{F}}$ & 6.811 & 8.379 & 6.226 & 7.032 \\
$B^{\mathrm{F}}$ & -133.3 & -195.4 & -88.89 & -35.52 \\
$\gamma^{\mathrm{F}}$ & 228.1 & 301.0 & 100.3 & 100.3 \\
$k_{0}^{\mathrm{F}}$ & 0.06721 & 0.04906 & 0.08069 & 0.07054 \\
$a^{\mathrm{F}-\mathrm{Li}}$ & 0.01079 & 0.01316 & - & 0.007949 \\
$b^{\mathrm{F}-\mathrm{Li}}$ & 0.002739 & 0.003098 & - & 0.001527 \\
$c^{\mathrm{F}-\mathrm{Li}}$ & 0.9816 & 0.9817 & - & 1.263 \\
$d^{\mathrm{F}-\mathrm{Li}}$ & 0.9906 & 1.056 & - & 2.028 \\
$a^{\mathrm{F}-\mathrm{Be}}$ & - & - & 0.01206 & 0.01527 \\
$b^{\mathrm{F}-\mathrm{Be}}$ & - & - & 0.002349 & 0.0003338 \\
$c^{\mathrm{F}-\mathrm{Be}}$ & - & - & 1.379 & 1.220 \\
$d^{\mathrm{F}-\mathrm{Be}}$ & - & - & 1.738 & 3.850 \\
$a^{\mathrm{F}-\mathrm{F}}$ & 0.000 & 0.000 & 0.0006233 & 0.001026 \\
$b^{\mathrm{F}-\mathrm{F}}$ & 0.003187 & 0.002477 & 0.001504 & 0.003598 \\
$c^{\mathrm{F}-\mathrm{F}}$ & 3.000 & 3.000 & 1.097 & 1.006 \\
$d^{\mathrm{F}-\mathrm{F}}$ & 0.7004 & 0.7384 & 1.556 & 1.239 \\
\hline
\end{tabular}

TABLE II: Parameters for the models used in the light scattering simulations of $\mathrm{LiF}$ and $\mathrm{BeF}_{2}$. Atomic units are used throughout. $\sigma$ values of 2.513 for $\mathrm{F}^{-}$and of 1.398 for both cations were used.

good agreement is evident, and the corresponding figure for the liquid has a similar quality, as is evident from the $A$ values in the table. 

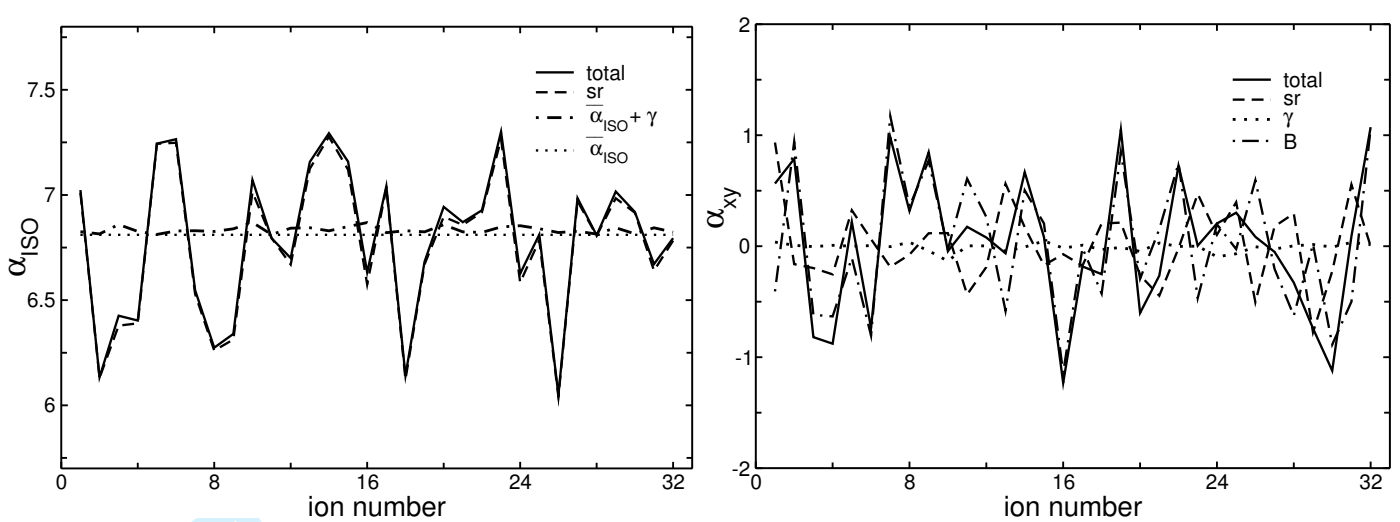

FIG. 2: The contributions of individual mechanisms to the overall polarizabilities: the left-hand panel shows the isotropic fluoride ion polarizabilities, whilst the right-hand panel shows an off-diagonal element, $\alpha_{x y}$. Values shown are for the last of five rocksalt configurations.

The optimal set of parameters for the polarizability model are given in table II. For the crystal, the parameters which emerge from the fitting procedure are very similar to those obtained from the previous quantum chemical calculations ${ }^{19,20}$. This is reassuring, as in the previous calculations the parameters pertaining to each mechanism from the fluctuating polarizability were obtained with independent, well-directed calculations; in the fits all of the mechanisms are lumped together. In particular, the $\gamma$ and $B$ polarizabilities were obtained from finite-field calculations with externally applied fields ${ }^{47}$. The parameters governing the short-range contributions $\left(a^{i j}, b^{i j}, c^{i j}\right.$ and $d^{i j}$ of equations 13 and 14) change very little between the solid and liquid phases, suggesting a degree of transferability of this short-range model. Considerable changes are seen however in the mean polarizability, $\bar{\alpha}$, the hyperpolarizabilities, $B$ and $\gamma$, and in the force constant $k_{0}$ (which is effectively a mean polarizability). The isotropic fluoride ion polarizability increases from an average of 6.81 a.u. in the solid to 8.38 a.u. in the liquid. This reflects the decrease in confining potential experienced by anions in the less dense liquid phase ${ }^{22}$ the majority of which find themselves in fouror five-coordinate environments compared to the six-coordinate octahedral environment of the solid. The larger values for the hyperpolarizabilities in the liquid phase reflect the same decrease in the strength of the confining potential. $k_{0}$ takes a value of 0.06721 in the solid phase, and 0.04906 in the liquid. If all short-range interactions are removed, these values imply gas-phase polarizabilities of $14.9 a_{0}^{3}$ and $20.4 a_{0}^{3}$ respectively. The first of these is not dissimilar to the calculated free-ion polarizability of $16 a_{0}^{3}$, and it is reassuring that in this limit, the model predicts a reasonable gas-phase polarizability.

Figure 2 shows the contributions of individual terms in the model to the overall polarizabilities. The lefthand panel shows the isotropic $\mathrm{F}^{-}$ion polarizabilities for the last of five rocksalt configurations. Their values result almost entirely from the short-range part of the model, with contributions from the $\gamma$-mechanism 
being dwarfed in comparison. Note that we have added a constant $\left(\bar{\alpha}^{\mathrm{F}}\right)$ to the $\gamma$ values to show their magnitude clearly on the same graph. The right-hand panel shows the anisotropic contributions resulting from the off-diagonal element, $\alpha_{x y}$, of these tensors. Once again, the $\gamma$-mechanism barely contributes, and the anisotropic components arise from a complicated interplay between the short-range and $B$ hyperpolarizability terms. Although not shown here, very similar results are seen for the liquid phase.

\section{B. Raman spectra of $\mathrm{LiF}$}

Calculations of the light scattering spectra of the melt and crystal phases of LiF have been undertaken using the models discussed above. We used the same quadrupole-polarizable interaction potential ${ }^{31}$ used to generate configurations for the ab initio calculations. Each simulation contained 512 ions, and was carried out in the $N V T$ ensemble at the density predicted by the potential under ambient pressure at the temperature of interest. For the crystal phase, the simulation was run at $298 \mathrm{~K}$, with data collection over a $0.363 \mathrm{~ns}$ trajectory of $1 \times 10^{6}$ timesteps. The liquid simulation was equilibrated for $1 \times 10^{5}$ timesteps at $1138 \mathrm{~K}$ and statistics calculated over $1 \times 10^{6}$ timesteps.

The full expression for the polarization induced by the external field (equations 5 and 6 ) should be solved iteratively in order to treat the dipole-induced dipole terms in a self-consistent manner. These terms are an additional source of fluctuations in the total polarizability $\Delta \Pi$ of the sample alongside those contained in $\left\{\boldsymbol{\alpha}^{i}\left(\left\{\mathbf{R}^{N}\right\}\right)\right\}$. Here we iterate equation 6 to first-order, and replace the instantaneous value of $\boldsymbol{\alpha}\left(\left\{\mathbf{r}^{N}\right\}\right)$ by $\bar{\alpha}$ so that the dipole-induced dipole term is calculated as if the ions respond with their average polarizability. The result is that these dipole-induced dipole terms behave as an additional contribution to the total polarizability

$$
\Pi_{\alpha \beta}=\sum_{i}\left[\alpha_{\alpha \beta}^{i,(s r)}\left(\left\{\mathbf{R}^{N}\right\}\right)+\alpha_{\alpha \beta}^{i,(\gamma)}\left(\left\{\mathbf{R}^{N}\right\}\right)+\alpha_{\alpha \beta}^{i,(B)}\left(\left\{\mathbf{R}^{N}\right\}\right)\right]-\sum_{i, j} \bar{\alpha}^{i} \bar{\alpha}^{j} T_{\alpha \beta}^{i j}
$$

and this is the expression we will use for the calculations of the spectrum from equation (1). This first-order dipole-induced dipole (DID) model will be adequate provided that the fluctuations in the polarizabilities are small compared to their mean values, otherwise the replacement of $\left\{\boldsymbol{\alpha}^{i}\left(\left\{\mathbf{R}^{N}\right\}\right)\right\}$ by $\bar{\alpha}$ will be jeopardized. If the ratio of the mean polarizability to the inverse cube of the interionic separation becomes large, higher order DID effects may become important. This could be significant when the cations as well as anions are polarizable, which is not the case in the materials studied here.

In atomic and van der Waals molecular liquids the DID terms make the dominant interaction-induced contribution to the light scattering spectrum ${ }^{50}$. Note that the first-order DID spectrum is then predicted to be purely depolarized (anisotropic), which follows from the traceless nature of $\mathbf{T}^{i j}$, and hence the depolar- 

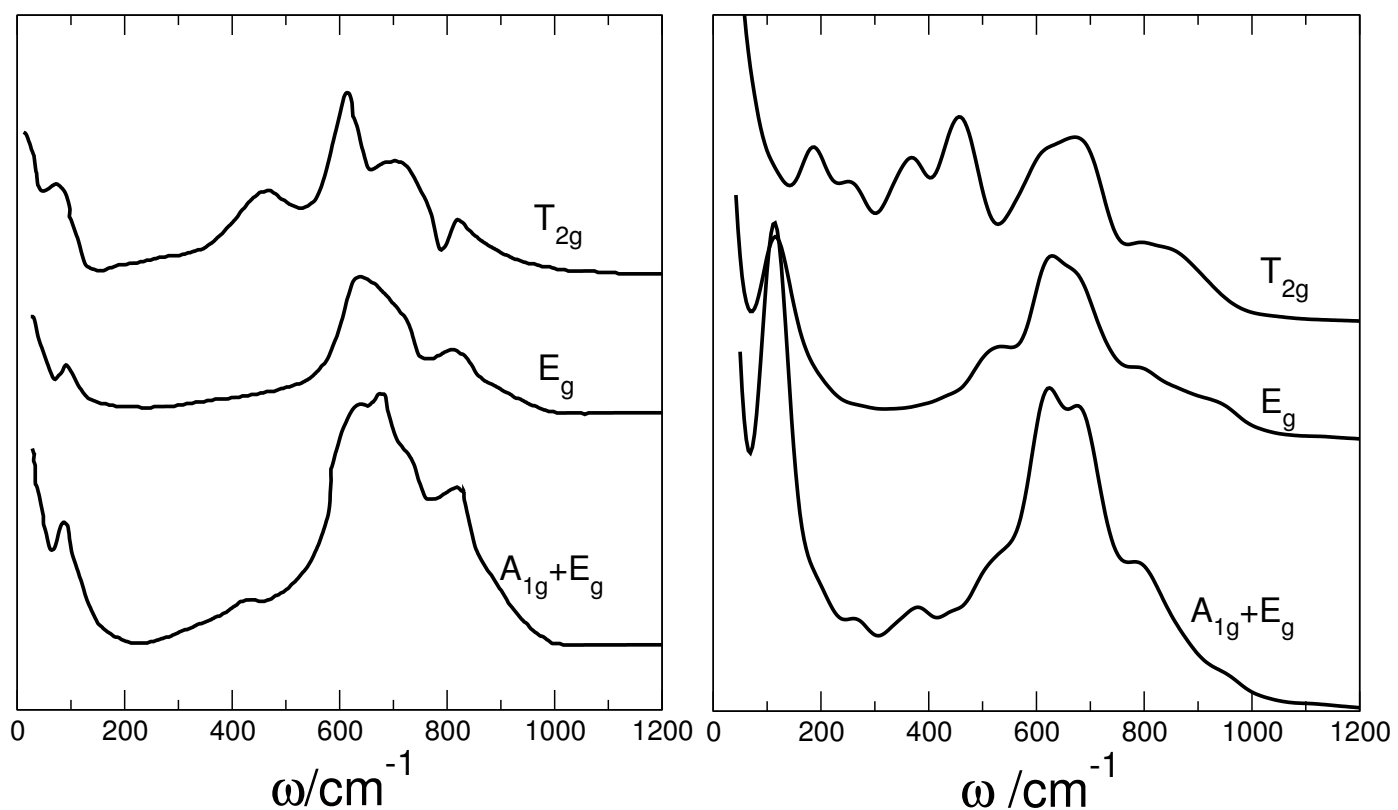

FIG. 3: The left-hand panel shows experimental spectra in three independent polarizations for crystalline $\mathrm{LiF}$ at 300 $\mathrm{K}$, redrawn from reference ${ }^{52}$. The $\mathrm{T}_{2 g}$ and $\mathrm{E}_{g}$ spectra have been increased by factors of 10 and 2 respectively. The right-hand panel shows corresponding spectra for crystalline LiF calculated from computer simulations at $298 \mathrm{~K}$, scaled in the same way as the experimental data.

ization ratio of the interaction-induced spectrum will be 0.75 . This is found to be the case in simple atomic liquids such as liquid Argon, but many of the alkali halide melts have been shown experimentally to possess significant isotropic components in their scattering, which points to the spectrum being dominated by the fluctuations contained in $\left\{\boldsymbol{\alpha}^{i}\left(\left\{\mathbf{R}^{N}\right\}\right)\right\}$.

\section{Results for the crystal}

Experimental spectra for crystalline $\mathrm{LiF}$ at $300 \mathrm{~K}$ in three polarizations have been published by Sharma et $a{ }^{52}$, and are reproduced in figure 3. These determine the three independent Raman spectra, resulting from fluctuations in the polarizability tensor of $A_{1 g}, E_{g}$ and $T_{2 g}$ symmetries for the cubic crystal. The scales for the $T_{2 g}$ and $E_{g}$ spectra have been amplified 10 and 2 times respectively, and the $A_{1 g}$ spectra occurs only in combination with the $\mathrm{E}_{\mathrm{g}}$ result as $\left(\mathrm{A}_{1 \mathrm{~g}}+\frac{4}{3} \mathrm{E}_{\mathrm{g}}\right)$. The shapes of these spectra reflect the form of the two-phonon density of states. Two phonons of equal and opposite wavevector may contribute to a spectrum if the distortion of the lattice they produce modulates the relevant component of the polarizability tensor $^{41}$. The number of such phonon pairs tends to peak in certain frequency regions (critical points) which lead to corresponding peaks in the spectrum. 
The spectra calculated from the simulations are shown in figure 3, right-hand panel. The agreement with experiment is rather good in all but the $\mathrm{T}_{2 \mathrm{~g}}$ case; this is by far the weakest spectrum (note the scale factor of ten). The $\mathrm{E}_{\mathrm{g}}$ spectrum compares very favorably; there is a large broad peak at around $625 \mathrm{~cm}^{-1}$ with a shoulder at high frequency, and a smaller peak at $800 \mathrm{~cm}^{-1}$. Both of these features are seen in the experimental spectrum. The simulated $\mathrm{E}_{\mathrm{g}}$ spectrum also shows a weak lower frequency peak at 540 $\mathrm{cm}^{-1}$, and a small bump at high frequency around $930 \mathrm{~cm}^{-1}$, which are not detected in the experimental spectrum. In the $\left(\mathrm{A}_{1 \mathrm{~g}}+\mathrm{E}_{\mathrm{g}}\right)$ spectrum, we see two additional peaks at 675 and $380 \mathrm{~cm}^{-1}$ arising from the $A_{1 g}$ contribution to the spectrum. For the first of these peaks, there is a clearly visible counterpart in the experimental spectrum, whilst for the second, we see a feature close by at around $410 \mathrm{~cm}^{-1}$. The calculated $\mathrm{T}_{2 \mathrm{~g}}$ spectrum is much weaker than either of the other two spectra, in agreement with experiment: it contains the broad features seen at 450,600-700, and $800 \mathrm{~cm}^{-1}$, but it lacks the sharp peak seen in the experimental spectrum at $\sim 600 \mathrm{~cm}^{-1}$, which makes the overall appearance of the calculated spectrum significantly different from the experimental one. It should be noted that Sharma $e t a l^{52}$ were able to reproduce these spectra with empirical lattice dynamics and polarizability models much better than we have on our first-principles, molecular dynamics basis.

A possible reason for missing this sharp feature is that we are not sampling the two-phonon density of states sufficiently finely. In an infinite crystal, there is a continuous range of phonon wavevectors from zero to the Brillouin zone boundary. The small size of our simulation cell $(4 \times 4 \times 4$ unit cells $)$ and the periodic boundary conditions used, mean that the simulation allows only a small number of possible wavevectors over this range. The number of possible phonon combinations which contribute to the two-phonon density of states is thus greatly reduced from the infinite crystal case. Nevertheless, we see good agreement with the broader features seen in the experimental spectra. It may be that these broader features arise from critical points to which a significant volume of the Brillouin zone contributes, and this is sufficiently well sampled by the limited set of wavevectors allowed in our simulation cell. The sharper peak appearing in the $\mathrm{T}_{2 \mathrm{~g}}$ spectrum, on the other hand, contains contributions from a much smaller volume of the Brillouin zone which is missed by our sampling scheme.

Bearing this limitation of calculating the two-phonon spectrum by MD in mind, we conclude that our calculation of the crystal LiF spectrum is satisfactory in that the distribution of intensities between different polarizations and between different bands in the spectrum seems to be generally very good. 


\section{Results for the liquid}

The Raman spectra of alkali halide melts are comparatively featureless. In figure 4, we show logarithmic plots of the isotropic $\left(I_{\mathrm{ISO}}\right)$ and anisotropic $\left(I_{\mathrm{ANI}}-\right.$ the average of the spectra calculated with the $\mathrm{E}$ and $\mathrm{T}$ components of the polarizability) spectra calculated as described above for the simulation of molten $\mathrm{LiF}$ at $1138 \mathrm{~K}$. These spectra are of similar shape to those obtained in our earlier work ${ }^{21}$ which have been compared in detail with the experimental spectra obtained by Papatheodorou et al. ${ }^{48}$. We will not repeat this comparison here, the lineshapes agreed well, except for the low frequency region of the depolarized spectrum, and the only significant point of departure concerned the depolarization ratio, $\rho(\omega)$. The experimental $\rho(\omega)$ reported in reference ${ }^{48}$ was essentially frequency independent and close to 0.1 . For the previous simulation ${ }^{21}$, overestimation of the anisotropic scattering was manifested in a depolarization ratio much greater than observed in experiment, and with a significant frequency dependence. The current theoretical predictions (appearing in the inset to figure 4) are much more in-line with the experimental observations; $\rho(\omega)$ is essentially constant with a value close to 0.18 . There is a clear improvement in our prediction of the shape of the depolarization ratio, though at first sight it would appear that the theoretical prediction is still somewhat too high. However, the light scattering spectra of LiF were later re-measured by the same group ${ }^{51}$, and although the spectral shapes measured were in excellent agreement with those measured previously, the relative intensity of the anisotropic to the isotropic was found to be higher in the new measurements. The new measurements will therefore result in an increase in the experimental depolarization ratio though its shape would be the same. Unfortunately for our purposes, these new figures have not been published. We attribute the improvement in the predicted depolarized spectrum to a better description of the cancelation between the contributions from the different mechanisms.

\section{The role of individual mechanisms}

It is of interest to see how each of the different mechanisms identified in the polarizability model affect the observed spectrum. Values for the zero-time correlation functions are given in table III, they determine the contribution to the total intensity. We show the auto-correlation functions of the polarizability fluctuations (per ion pair) associated with a given mechanism, $A\left(=s r, B, \gamma\right.$ or DID), for example, $\left\langle\left|\Delta \Pi_{\mathrm{ISO}}^{A}\right|^{2}\right\rangle$ (denoted $I_{\text {ISO }}^{A}$ ).

The auto-correlation values give an idea of the importance of that mechanism. We can see therefore that the short-range term is the most important contributor to the isotropic scattering, and dominates the $\gamma$ contribution. For the anisotropic scattering, large contributions are seen from three of the four mechanisms, 

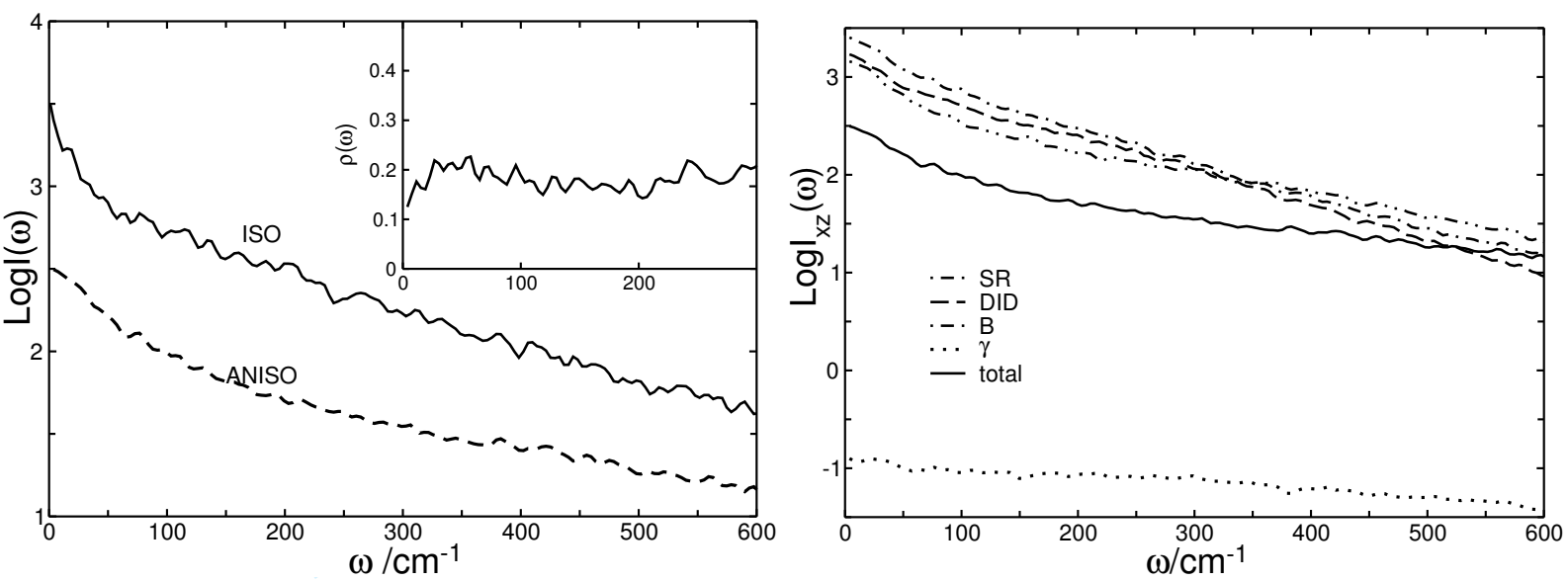

FIG. 4: The simulated isotropic and anisotropic light scattering lineshapes for $\mathrm{LiF}$ at $1138 \mathrm{~K}$ are shown in the left-hand panel; also shown in the inset is the simulated depolarization ratio. Right-hand panel: Contributions of the individual mechanisms to the anisotropic scattering in liquid LiF. The spectra associated with the short-range, DID, $B$ and $\gamma$ contributions to the anisotropic scattering are compared with the total spectrum for liquid LiF.

\begin{tabular}{|lccccc|}
\hline & $\mathrm{LiF}^{2}$ & $\mathrm{BeF}_{2}$ & & $\mathrm{LiF}$ & $\mathrm{BeF}_{2}$ \\
\hline$I_{\mathrm{ISO}}^{s r}$ & 3.790 & 5.348 & $I_{\text {ISO }}^{\gamma}$ & 0.003 & 0.106 \\
$I_{\text {ANI }}^{s r}$ & 2.487 & 1.744 & $\kappa_{\text {ANI }}^{s r-\mathrm{DID}}$ & -0.78 & -0.97 \\
$I_{\mathrm{ANI}}^{\gamma}$ & 0.001 & 0.067 & $\kappa_{\text {ANI }}^{s r-B}$ & -0.77 & -0.89 \\
$I_{\text {ANI }}^{B}$ & 4.179 & 4.152 & $\kappa_{\text {ANI }}^{\text {DID }-B}$ & -0.92 & -0.42 \\
$I_{\text {ANI }}^{\text {DID }}$ & 2.986 & 2.668 & & & \\
\hline
\end{tabular}

TABLE III: Scattering intensities by mechanism for liquids $\mathrm{LiF}$ and $\mathrm{BeF}_{2}$; polarizability fluctuations are given per formula unit for each system. In the second column, the $\kappa$ values indicate the strength of correlations between the most significant mechanisms.

with the $\gamma$ term making a negligible contribution. The DID term is appreciable in these simulations, much more so than was seen in the previous simulation results. This can be attributed to the use of a larger fluoride ion polarizability (taken from DFT calculations on the liquid phase, rather than the crystal value).

The magnitudes of the cross-correlations are reported in table III in terms of the parameter $\kappa^{A-B}$

$$
\kappa^{A-B}=\left\langle\Delta \Pi^{A} \Delta \Pi^{B}\right\rangle /\left[\left\langle\left|\Delta \Pi^{A}\right|^{2}\right\rangle\left\langle\left|\Delta \Pi^{B}\right|^{2}\right\rangle\right]^{\frac{1}{2}}
$$

whose magnitude is governed by the Schwartz inequality to lie between +1 and -1 , with the limits reached if the polarizability fluctuations due to mechanisms $A$ and $B$ are perfectly correlated or anti-correlated. In LiF we can see that there is a very high degree of (anti-)correlation between the DID and $B$ terms, which means that their net contribution to the integrated intensity will be much smaller than would be predicted from their 
auto-correlation alone. There are similar, weaker anti-correlations between the $s r$ and DID terms, and the $s r$ and $B$ terms. The correlation functions involving $\gamma$ are too small to merit consideration. The physical reasons for these correlations was discussed previously on the basis of a rather more ad hoc version of the model $^{20}$. Although the different terms in the model arise from well-defined distinct physical sources, the very high degree of correlation between them we now find with the ab initio-parameterized model suggests that we should reconsider whether they ought to be represented separately. We will return to reconsider this issue at the end of the article.

The overall effect of these cross-correlations is to make the attribution of the anisotropic scattering to a single mechanism very difficult. Indeed, the amplitude of the total polarizability fluctuation is only around one-quarter of the fluctuations seen in the auto-correlation functions. In the right-hand panel of figure 4 we show anisotropic spectra associated with the individual interaction-induced polarizability mechanisms, which we have called "subspectra" in previous works 9 . The spectra of the cross-correlations tend to behave as a mixture of their parents. Also shown for comparison on the same intensity scale is the total spectrum; its intensity is substantially less than several of the component spectra due to the anti-correlations discussed above.

The $\gamma$ contribution appears to be negligible, and we confine our attention to the three remaining terms. We first note the similarity between the shape of the $\Delta \Pi^{\mathrm{DID}}$ and $\Delta \Pi^{B}$ spectra, and the difference in lineshape from the short-range spectra. These results suggest that the positional fluctuations responsible for most of the $\Delta \Pi^{B}$ intensity are longer range than those which show up in the short-range spectra, and that this mechanism appears to couple to the same set of fluctuations seen in the DID spectra. In the region of $0-350 \mathrm{~cm}^{-1}$, the high degree of anti-correlation between the DID and $B$ terms seems to almost completely cancel the influence of these terms on the lineshape, and the shape of the total spectrum is very similar to that due to the short-range term alone. In practice, except the highest frequencies, the anisotropic line shape may be regarded as a property of $\Delta \Pi^{s r}$ reflecting the near-neighbor dynamics in the melt.

\section{RESULTS: SIMULATIONS OF $\mathrm{BEF}_{2}$}

\section{A. Parameterization of the model for $\mathrm{BeF}_{2}$}

Using a quadrupole-polarizable interaction potential ${ }^{39}$ we carried out an equivalent parameterization of the light scattering model for liquid $\mathrm{BeF}_{2}$. DFT calculations of the ionic polarizabilities were performed on three liquid configurations, each containing 72 ions, which were taken from simulations of the liquid phase at $1300 \mathrm{~K}$. The $\mathrm{F}^{-}$ion polarizability $\bar{\alpha}^{\mathrm{F}}$ extracted from these calculations are smaller than those seen in 
liquid $\mathrm{LiF}$, with an average value of $6.23 a_{0}^{3}$. The results of the fitting procedure are shown in table I, and the parameters obtained are given in table II. The anion-cation short-range parameters $a^{\mathrm{F}-\mathrm{Be}}$ and $b^{\mathrm{F}-\mathrm{Be}}$ are quite similar to the corresponding Li-F values, whilst the parameters $c^{\mathrm{F}-\mathrm{Be}}$ and $d^{\mathrm{F}-\mathrm{Be}}$ are slightly larger. This is consistent with a short-range effect of comparable magnitude, but which decays more rapidly with ionic separation due to the smaller size of the $\mathrm{Be}^{2+}$ ion. The hyperpolarizabilities of the $\mathrm{F}^{-}$ion are considerably smaller than those obtained in the LiF case. This may be a consequence of the greater compression of the $\mathrm{F}^{-}$ion, as witnessed by the reduction in $\bar{\alpha}^{\mathrm{F}}$, but it may also be a suggestion that the fitting process is not differentiating the different terms sufficiently well. In the LiF fits we set the initial values of the hyperpolarizabilities to the values which had been found in the previous embedded cluster calculations ${ }^{47}$ and found good fits without a substantial change in these values: in $\mathrm{BeF}_{2}$ the hyperpolarizability values consistently moved to smaller values.

The fit quality is again good, showing that, despite the higher cation charge and the less symmetrical coordination environment around the anions in $\mathrm{BeF}_{2}$ compared to $\mathrm{LiF}$, the model is capable of reproducing the DFT-calculated polarizabilities. The fluoride ions in liquid $\mathrm{BeF}_{2}$ are predominantly bridging between adjacent $\mathrm{BeF}_{4}^{2-}$ tetrahedra, with two nearest-neighbor $\mathrm{Be}^{2+}$ ions forming a bent $\mathrm{Be}-\mathrm{F}-\mathrm{Be}$ bond.

\section{B. Light scattering in pure $\mathrm{BeF}_{2}$}

The Raman spectrum of liquid $\mathrm{BeF}_{2}$ was calculated at $1300 \mathrm{~K}$. The simulations were carried out with both quadrupole and dipole polarizable interaction potentials ${ }^{39}$, with similar results obtained in both cases. The simulations were equilibrated over several nanoseconds (the structural relaxation time at $1300 \mathrm{~K}$ is over $100 \mathrm{ps)}$ and the spectra were obtained from trajectories of approximately $200 \mathrm{ps}$. In figure 5, we show the experimental polarized and depolarized spectra for the room temperature glass measured by Galeener et al. ${ }^{34}$, together with the corresponding spectra calculated from the simulations of the liquid. The Raman spectrum of $\mathrm{BeF}_{2}$ is much more structured than that of $\mathrm{LiF}$ due to the persistence of quasi-molecular ionic complexes, like $\mathrm{BeF}_{4}^{2-}$, on much longer timescales than the Raman observation ${ }^{32,40}$. We were unable to find an experimental spectrum for the liquid phase but, to the extent that there is no major shift in the equilibria governing the formation of these species due to the change of temperature, we can expect the liquid and glassy spectra to be comparable.

The main features of the experimental Raman spectra are predicted well by the simulation. There is a strong highly polarized feature at $282 \mathrm{~cm}^{-1}$, with several shoulders on both the high and low frequency side, whose origin has been ascribed to a symmetric-stretching type motion of the bridging fluoride ions ${ }^{34}$. A highly polarized peak with similar sidebands is present in the simulated spectrum at around $300 \mathrm{~cm}^{-1}$; 

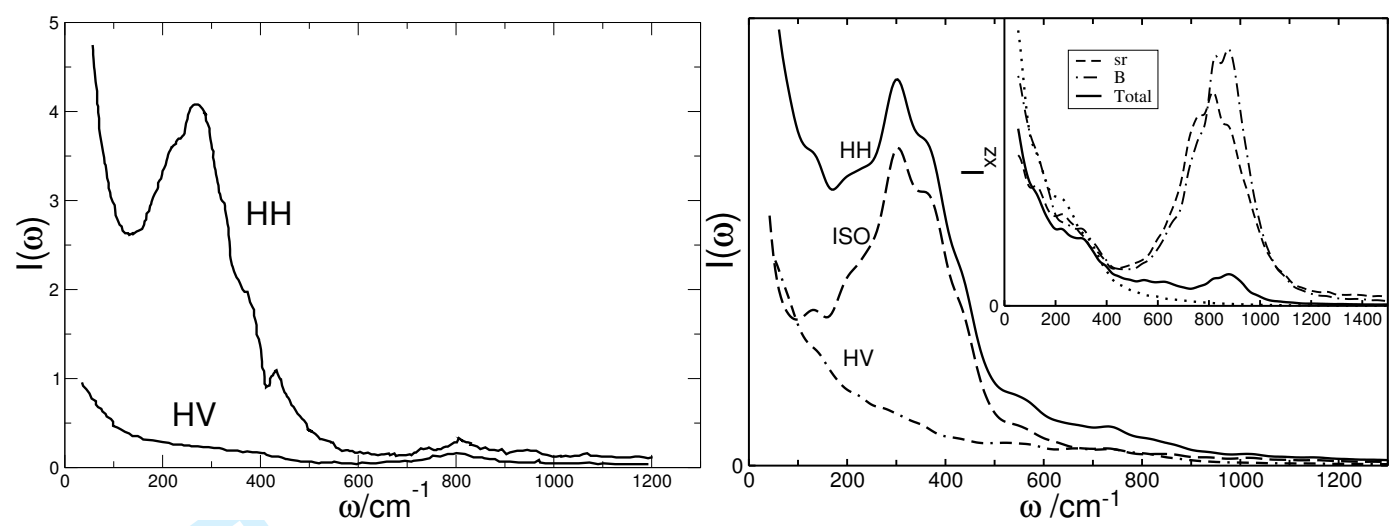

FIG. 5: The polarized and depolarized Raman spectra of vitreous $\mathrm{BeF}_{2}$ at room temperature, redrawn from ${ }^{34}$, are shown in the left-hand panel: the sharp glitch at about $410 \mathrm{~cm}^{-1}$ is an experimental artifact. In the right-hand panel the corresponding spectra from simulations of liquid $\mathrm{BeF}_{2}$ at $1300 \mathrm{~K}$ are shown, together with the isotropic spectrum. In the inset the contributions of the different scattering mechanisms to the depolarized spectrum are shown, note the strong cancelation of the $s r$ and $B$-tensor mechanisms in the region of $800 \mathrm{~cm}^{-1}$

it shows a shoulder at $361 \mathrm{~cm}^{-1}$ on the high frequency side of the main polarized peak, which arises entirely from the isotropic scattering. The latter appears at exactly the same frequency as the smaller of the two peaks in the simulated infra-red absorption spectra $^{39}$. A shoulder with the same shift from the main polarized band is also seen in the experimental Raman spectrum and Galeener et $a l^{34}$ noted that the frequency of this shoulder coincided with that of the smaller peak in the experimental IR spectrum ${ }^{34}$. At higher frequencies, between 700 and $900 \mathrm{~cm}^{-1}$, two or three weaker, broad and much less highly polarized lines are also seen. Corresponding depolarized bands are seen in the experimental spectrum, at slightly higher frequencies.

We will discuss the origin of these features in more detail below, after we have discussed the spectra of the $\mathrm{LiF} / \mathrm{BeF}_{2}$ mixtures.

\section{The role of individual mechanisms}

The values for the zero-time correlation functions are given in table III, alongside those for LiF. The short-range term is again the dominant contributor to the isotropic scattering, and far outweighs the $\gamma$ term. The short-range, DID and $B$ mechanisms all have substantial auto correlations which suggest that the make important contributions to the anisotropic scattering. There is however, a very large degree of anticorrelation between these terms, so that their net contribution to the scattering intensity is greatly reduced and it is difficult to associate specific features in the total spectrum with a single mechanism. 
This issue is highlighted in the inset to the right-hand panel of figure 5, which shows the anisotropic spectra associated with the individual interaction-induced polarizability mechanisms, as well as the total spectrum. It can be seen that the $B$ and $s r$ mechanisms both have substantial peaks at about $800 \mathrm{~cm}^{-1}$, this is the region of asymmetric stretching vibrations of the $\mathrm{BeF}_{4}$ tetrahedra. However, because of the anti-correlation between the $B$ and $s r$ contributions, the total spectrum shows only a weak peak at this frequency. From one perspective, this finding is a considerable success since the feature is indeed weak in the experimental spectrum. On the other hand, it is not desirable to have the observable appear as a difference between two large terms, resulting in large errors as a consequence of small errors in the individual terms. The DID mechanism may contribute to the scattering in the lower frequency regions (less than $500 \mathrm{~cm}^{-1}$ ), but does not seem to show up the vibrational features of the coordination polyhedra in the same way as the other mechanisms.

\section{LIF-BEF 2 MIXTURES}

\section{A. Parameterization of the model for $\mathrm{LiF}_{-} \mathrm{BeF}_{2}$ mixtures}

It is very desirable that the polarizability model is tranferable between the pure materials and their mixtures - this should be so if the model contains a good representation of the physics governing the interaction-induced contributions to the polarizabilities of the ions. We have noted above that the parameters in the model seem to be sensibly related between $\mathrm{LiF}$ and $\mathrm{BeF}_{2}$, though we have seen larger than expected changes in some values, notably the hyperpolarizabilities. In order to see if we can produce a good, transferable compromise, a polarizability model for the $\mathrm{LiF}_{-} \mathrm{BeF}_{2}$ mixtures was obtained by fitting to the $a b$ initio polarizabilities for the pure liquid $\mathrm{LiF}$ and $\mathrm{BeF}_{2}$ simultaneously. In the past, we have shown that a very good description of the interionic interactions in the mixture can be obtained by fitting a single interaction potential to $a b$ initio data on both pure phases. The results of the fitting of the polarizability model are shown in table I, and the parameters obtained are given in table II.

The quality of the fit is particularly good for the isotropic polarizability, and is comparable to that obtained in the case of pure $\mathrm{BeF}_{2}$. This is remarkable as the data in table II shows that the mean polarizabilities of the $\mathrm{F}^{-}$ions in liquid $\mathrm{LiF}$ and $\mathrm{BeF}_{2}$ differ quite considerably. The short-range term alone dominates the isotropic polarizability. Using separate short-range parameters to model the effects of the neighboring $\mathrm{Li}^{+}$ and $\mathrm{Be}^{2+}$ ions, it has been possible, within a single model to reproduce the difference in average polarizability between these two liquid phases and also the fluctuations. The quality of the fit to the anisotropic components of the polarizability is less satisfactory, which probably reflects the difficulty of representing 
the hyperpolarization effects in both systems with the same hyperpolarizabilities. One final point concerning the model is the value used for the average $\mathrm{F}^{-}$polarizability which determines the strength of the DID effects. In this work, we have taken its value to be the average of the DFT calculated polarizability of the fluoride ion in the liquid phases of $\mathrm{LiF}$ and $\mathrm{BeF}_{2}$.

\section{B. Light scattering in $\mathrm{LiF}-\mathrm{BeF}_{2}$ mixtures}

We performed simulations of the Raman scattering from three $\mathrm{LiF}_{-} \mathrm{BeF}_{2}$ mixtures containing 75,67 and $50 \% \mathrm{LiF}$ at temperatures of $873 \mathrm{~K}, 873 \mathrm{~K}$ and $1073 \mathrm{~K}$, respectively. The trajectories were continuations of those used to study the conductivity and viscosity of these systems ${ }^{32}$, and have already been well equilibrated. The simulations of the light scattering spectra were performed over one million MD steps approximately $360 \mathrm{ps}$ - on samples of roughly 500 ions.

In figure 6, we show an example of an experimental Raman spectra for the mixture of composition 60-40 $\% \mathrm{LiF}_{-} \mathrm{BeF}_{2}{ }^{38}$, which shows the general form exhibited by each of these spectra. It was also noted that the effect of temperature on these $\mathrm{LiF}-\mathrm{BeF}_{2}$ spectra was negligible. The main features are a broad, polarized peak at $525 \mathrm{~cm}^{-1}$, and much weaker, depolarized bands at $\sim 800 \mathrm{~cm}^{-1}, 383 \mathrm{~cm}^{-1}$ and $220 \mathrm{~cm}^{-1}$. As the melt composition is changed, similar features are observed until the $\mathrm{BeF}_{2}$ mole fraction exceeds $50 \%$ though the bands broaden and shift. For higher concentrations the spectrum progressively changes shape and evolves into the form of the network spectrum also seen in $\mathrm{SiO}_{2}$ and $\mathrm{GeO}_{2}$. In particular the strong polarized peak appearing at about $525 \mathrm{~cm}^{-1}$ in the relatively dilute mixtures shifts progressively to lower frequencies. In the limiting case of pure molten $\mathrm{BeF}_{2}$, it converges to the previously discussed $282 \mathrm{~cm}^{-1}$ band $d^{34}$.

In the right-hand panel of figure 6 , we show the polarized, depolarized and isotropic scattering of the mixtures predicted in our simulations at the 67-33 composition, close to the composition of the experimental spectrum. The agreement between simulation and experiment is good, though the intensity of the broad depolarized band close to $800 \mathrm{~cm}^{-1}$ seems to be underestimated. The reason for this can be seen in the inset to the right-hand panel of the figure, where we show the spectra associated with the $B$ and $s r$ mechanisms together with the total anisotropic spectrum. Both "subspectra" contain strong broad peaks at $800 \mathrm{~cm}^{-1}$ but, because of the anti-correlation between the two mechanisms, to which we have drawn attention above, the two contributions almost perfectly cancel out in the total spectrum. It is clear then that the relevant vibrational motion is present in the simulations, and that it can couple to the polarizability, but that the degree of anti-correlation between the different mechanisms is overestimated in the polarizability model we have used. Spectra of similar appearance were obtained at the other compositions studied. 


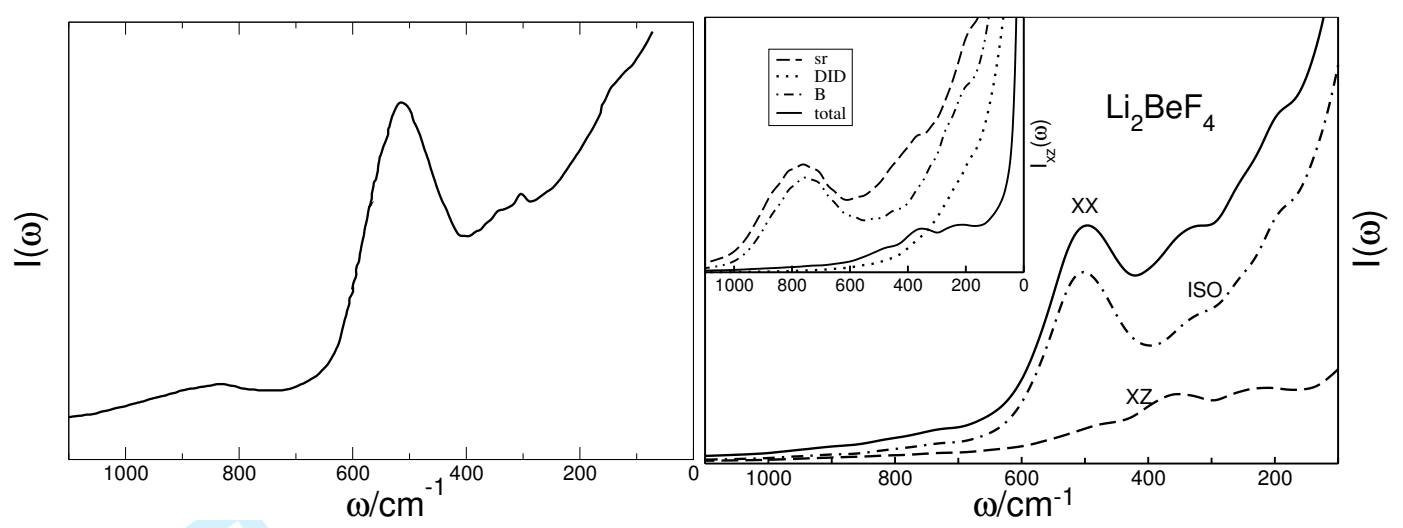

FIG. 6: Experimental Raman spectrum of molten $\mathrm{LiF}_{-} \mathrm{BeF}_{2}(60-40 \mathrm{~mol} \%)$ from reference ${ }^{38}$ are shown in the lefthand panel: the sharp feature at about $350 \mathrm{~cm}^{-1}$ is an experimental artifact. In the right-hand panel we show the polarized, depolarized and isotropic spectra calculated from the simulations. In the inset we show the contributions of the separate mechanisms to the depolarized spectrum: note the strong cancellation of the $s r$ and $B$-tensor mechanisms resulting in a very low intensity for the strong feature at $800 \mathrm{~cm}^{-1}$ in the calculated spectrum.

\begin{tabular}{|cccc|}
\hline & $75-25$ & $66-34$ & $48-52$ \\
& $873 \mathrm{~K}$ & $873 \mathrm{~K}$ & $1073 \mathrm{~K}$ \\
\hline$A_{1}$ & $532(550)$ & $533(540)$ & $545(480)$ \\
$E$ & $230(235)$ & $243(-)$ & $235(-)$ \\
$T_{2}$-stretch & $770(795)$ & $769(790)$ & $795(820)$ \\
$T_{2}$-bend & $359(375)$ & $355(365)$ & $355(-)$ \\
\hline
\end{tabular}

TABLE IV: Vibrational densities of states for the tetrahedral normal modes in $\mathrm{LiF}^{-\mathrm{BeF}_{2}}$ melts. The peaks of experimentally observed Raman peaks, where discernible, are given in brackets. Note that the good correspondence between the experimental spectrum and isolated tetrahedral ion picture at low $\mathrm{BeF}_{2}$ concentrations progressively degrades as the network forms.

The structures of the $\mathrm{LiF}-\mathrm{BeF}_{2}$ mixtures are dominated by the strong tendency of the $\mathrm{F}^{-}$ions to form long-lived tetrahedral complexes with $\mathrm{Be}^{2+}$. In the more dilute (in $\mathrm{BeF}_{2}$ ) mixtures, independent $\mathrm{BeF}_{4}^{2-}$ ions may form, and the Raman spectra are dominated by the characteristic bands expected for tetrahedral molecular ions which behave as independent scattering centres. In this régime, we can compare the positions of the Raman bands with the peaks of the density of states of the vibrational normal modes of these molecular ions, which can be calculated by the method of Pavlatou et $a l^{53}$. In table IV we compare the positions of these maxima with the positions of the Raman band peaks reported in ${ }^{38}$. For the most dilute mixture (75:25) there is a good correspondence between the normal mode frequencies and the experimental Raman band positions, the latter appear at slightly higher frequency than the simulations. The polarization 
characteristics are in accord with the symmetries of the normal modes. The correspondence is still quite good at 66:34; this is the limiting composition $\left(\mathrm{Li}_{2} \mathrm{BeF}_{4}\right)$ at which a picture of independent $\mathrm{BeF}_{4}^{2-}$ ions might be appropriate, at higher concentrations these tetrahedral units must progressively link together to form more and more complex oligomers $\left(\mathrm{Be}_{2} \mathrm{~F}_{7}^{3-}, \mathrm{Be}_{3} \mathrm{~F}_{10}^{4-}\right.$, etc. $)$, eventually leading to the formation of a complete tetrahedrally coordinated network in pure $\mathrm{BeF}_{2}$. This process was detailed in reference ${ }^{32}$. The oligomer vibrations involve coupling of the $\mathrm{BeF}_{4}^{2-}$ units so that the vibrational bands seen in the Raman (and infrared) spectrum occur at different frequencies to those calculated from the densities of states of the individual $\mathrm{BeF}_{4}^{2-}$ centres.

The simulated Raman spectra track the changes seen in the experimental spectra caused by this coupling. The most distinctive change involves the lowering of the frequency of the main polarized band from $\sim 550 \mathrm{~cm}^{-1}$ for the isolated $\mathrm{BeF}_{4}^{2-}$ ion in the $75: 25$ mixture to the $\sim 282 \mathrm{~cm}^{-1}$ in the pure melt. This associated with a change in the character of the relevant vibration from a stretch of a single Be-F bond to a complex coupled vibration of the Be-F-Be linkages which make up the network. Each of the simulated spectra for the $\mathrm{LiF}-\mathrm{BeF}_{2}$ mixtures show a single, broad and polarized peak such as that seen in experiment: for the mixture of composition $75-25 \mathrm{~mol} \% \mathrm{LiF}^{-B_{e} F_{2}}$, this peak is observed at $504(550) \mathrm{cm}^{-1}$, and shifts to lower frequency as the $\mathrm{BeF}_{2}$ content of the melt is increased. There is a small shift to $503(540) \mathrm{cm}^{-1}$ at $67-33 \mathrm{~mol} \%$, followed by a much larger shift to $443(480) \mathrm{cm}^{-1}$ at the 50-50 composition, the peaks in the experimental spectra are given in brackets ${ }^{38}$. Although the frequencies at which we observe this peak are consistently $\sim 40 \mathrm{~cm}^{-1}$ too low, the simulations predict rather well the shift in its position with changing composition of the melt. As we have already seen there is a good correspondence between the shape and position of this polarized band in the pure melt.

The formation of the network has a more subtle effect on the bands seen in the depolarized spectrum. As shown in figure 6 and table IV the broad band at $\sim 800 \mathrm{~cm}^{-1}$ in the dilute solutions may be associated with the density of states of the $\mathrm{T}_{2}$ stretching vibrations of the $\mathrm{BeF}_{4}^{2-}$ units, which are active in the infrared and Raman (depolarized). Weak depolarized features are observed in the Raman and infared spectra of the pure melt in the same frequency range, and whilst the overall character of the ionic motion which is responsible for the spectra remains the same, several sub-bands form. When the network forms, these vibrations are no longer independent and we must take into account the equivalent of the " $k=0$ selection rule" of the spectroscopy of crystals - i.e. only the vibrations of different bonds which make in-phase contributions to the polarizability and dipole moment are seen in the Raman and infrared spectra. Galeener et al. ${ }^{34}$ noted three features in the experimental spectra at frequencies of 745,810 , and $940 \mathrm{~cm}^{-1}$, the first of these appears at the position of the high-frequency peak in the infra-red absorption spectra. We also see in the $B$ and $s r$ subspectra the broad density of states band (right-hand panel of figure 6) breaking up into three components 
(right-hand panel of figure 5) with the lowest frequency component the infrared active one. It is possible to recognize similar features in the spectra of long wavelength charge-density waves in the simulations ${ }^{28}$.

\section{DISCUSSION}

We have described a practical process for the calculation of a Raman spectrum from first principles which can be applied to molten systems; this has been made possible by the ability to calculate the polarizabilities of individual ions in a general condensed-phase environment ${ }^{26}$. No experimental information was used in the simulations of the Raman spectra we have illustrated, and their comparison with the available experimental data at least suggests that it would be worthwhile to further refine the procedure. This depended on the introduction of specific functional forms for the dependence of the polarizability and the interaction potential on the ionic positions. The representation of the interaction potential has been developed systematically over a number of years and shown to reproduce other properties of the materials we have studied here quite accurately. In this work, those aspects which reflect on the quality of the underlying potential have performed well. Except for the $\mathrm{T}_{2 \mathrm{~g}}$ component of the LiF crystal spectra, which is a very demanding test, the frequencies of the experimentally observed Raman peaks have been reproduced quite accurately as well as the evolution of the $\mathrm{BeF}_{2}: \mathrm{LiF}$ spectra from the dilute solution to the network melt. Critical attention should therefore focus on the representation of the fluctuating polarizability.

The model used for the polarizability was that suggested by calculations of the polarizabilities of ions embedded in distorted crystal lattices ${ }^{20}$ on relatively weakly interacting, high coordination number ionic systems like alkali halides. The model involves the introduction of several different mechanisms each of which could be independently recognised in that context. In the present work, the isotropic polarizability fluctuations appear to have been modeled very successfully, in all cases it proved straightforward to fit the fluctuating polarizabilities coming from the $a b$ initio calculations and the isotropic contributions to the spectra have been reproduced well, judging by the comparison with experiment. In the materials studied, however, only the short-range mechanism has made a significant contribution, so the representation has, in this case, turned out to be rather simple. The parameters appearing in the Drude-inspired representation of these terms have changed from one material to another in a sensible way. The ability to represent the isotropic polarizability reliably is significant because most of the structural information about coordination structures etc. is derived from the isotropic contributions to the polarized experimental spectra ${ }^{2}$.

Several observations suggest that the representation of the anisotropic components of the polarizability tensor has been less successful, it appears that the multi-mechanism representation may be more complicated than is necessary or desirable for the melt spectra. In the anisotropic (depolarized) spectra we have 
calculated here, three mechanisms have been found to play an important role. Although we have been able to fit the $a b$ initio data, the parameter values have differed from one material to another in an unsystematic way. In the calculated spectra we have seen that there may be a very strong (destructive) interference between the different terms, which leads to errors through almost complete cancelation. We have also seen that different mechanisms have similar subspectra, especially the $s r$ and $B$-tensor. These strong correlations between different terms suggests that they are all being triggered by the same types of fluctuation in the fluid. This is, at first sight, surprising since the $B$-tensor, DID (and $\gamma$ ) terms all involve long-ranged functions of the interatomic fluctuations, whereas the $s r$-term with which they interfere involves functions which only extend over one or two ionic diameters. The conclusion is however, that the inclusion of the explicitly long-ranged contributions to the polarizability may be unnecessary and that the fitting procedure and the subsequent calculation of the spectra would be more straightforward if a single, short-ranged form were introduced which incorporated all the effects currently separated in the $s r, B$-tensor and $\gamma$ forms. We note that Pasquarello ${ }^{4}$ and Scandolo ${ }^{8}$ and co-workers have shown how (short-ranged) bond-polarizability models may represent the calculated polarizability fluctuations well in materials like $\mathrm{SiO}_{2}$, and this suggests that we should explore the use of such models in future extensions of this work.

\section{Acknowledgments}

The work was supported by EPSRC grant GR/R39138. The electronic structure calculations were performed on the IBM Bluegene supercomputer at the Edinburgh Parallel Computer Centre and we are grateful to the staff there for facilitating our use of the machine. 
1 Stone, A. J. Theory of Intermolecular Forces. Oxford University Press, Oxford, (1996).

2 Papatheodorou, G.N. and Yannopoulos, S.N. page 47-106 in Molten Salts: from Fundamentals to Applications, Ed. Gaune-Escard, M., Nato Science Series, Series II, vol. 52, (2002).

3 Windisch, C.F. and Risen, W.M. J. Non-Cryst. Solids, 48, 307 (1982)

4 Umari, P. and Pasquarello, A. Phys. Rev. Lett., 95137401 (2005)

5 Gilbert, B., Robert, E., Tixhon, E., Olsen, J., Ostvold, T. Inorg. Chem.,1996, 35, 4198, J. Phys. Chem. B 1997, $101,9447$.

6 Kalampounias, A.G., Yannopoulos, S.N., and Papatheodorou, G.N., J. Chem. Phys., 125, 164502 (2006).

7 McMillan, P.F., Poe, B.T., Gillet, P. and Reynard, B., Geochim. Cosmochim. Acta,58 3653 (1994)

8 Liang, Y.F., Miranda, C.R. and Scandolo, S., J. Chem. Phys., 125, 194524 (2006).

9 Glover, W.J. and Madden, P.A., J. Chem. Phys. 121, 7293 (2004)

10 Berne, B.J. and Pecora, R., Dynamic Light Scattering, (Wiley, New York, 1976)

11 d'Alessio, L., Pietrucci, F., and Bernasconi, M., J. Phys. Chem. Solids, 68, 438 (2007).

12 Putrino, A. and M. Parrinello, M., Phys. Rev. Lett., 88, 176401 (2002).

13 Giacomazzi, L. and Pasquarello, A. J. Phys. Condens. Matt. 19, Art 415112 (2007).

14 Ribeiro, M. C. C., Wilson, M. and Madden, P.A., J. Chem. Phys. 110, 4803 (1999).

15 Stillinger, F.H. and Webber, T.A., Phys. Rev. A, 25, 978 (1982)

16 Mazur, P. Adv. Chem. Phys., bf 1, 309 (1958).

17 Felderhof, B.U., Physica, 76, 486 (1974).

18 Madden, P.A., and Kivelson, D. Adv. Chem. Phys, 56, 467 (1984).

19 Fowler. P.W. and Madden, P.A., Phys. Rev. B, 31, 5443 (1985).

20 Madden., P.A. and Board, J.A. J. Chem. Soc. Faraday Trans. II, 83, 1891 (1987).

21 Madden, P. A., O’Sullivan, K., Board, J.A. and Fowler, P.W. J. Chem. Phys., 94(2), 918 (1991).

22 Fowler. P.W. and Madden, P.A., Phys. Rev. B, 29, 1035 (1984).

23 Madden, P.A., Wilson, M. and Hutchinson, F. J. Chem. Phys., 120(14), 6609 (2004).

24 Akdeniz, Z. and Madden, P.A. J. Phys. Chem. 110, 6683 (2006).

25 Jemmer, P., Wilson, M., Madden, P.A. and Fowler, P.W., J. Chem. Phys. 111, 2038 (1999)

26 Heaton, R.J., Madden, P.A., Clark, S.J. and Jahn, S., J. Chem. Phys. 125, 144104 (2006).

27 Pyper, N.C., Adv. Sol. Stat. Chem., 2, 223 (1991).

28 Heaton, R.J., Ph.D. Thesis, University of Edinburgh, 2007.

29 Laio, A., Bernard, S., Chiarotti, G.L., Scandolo, S., and Tosatti, E. SCIENCE 287 1027, (2000)

30 Aguado, A., Bernasconi, L., Jahn, S. and Madden, P.A., Faraday Disc. 124, 1712003.

31 Madden, P.A., Heaton, R.J. Aguado, A. and Jahn, S. J. Mol. Struct. THEOCHEM 771, 9 (2006).

32 Salanne, M., Simon, C. Turq, P., Heaton, R.J. and Madden, P.A. J. Phys. Chem. 110, 11461 (2006)

33 Müller, U. "Inorganic Structural Chemistry", (Wiley, Chichester, 1993) 
34 Galeener, F. L., Leadbetter, A. J. and Stringfellow, M. W.. Phys. Rev. B, 27, 1052 (1983).

35 Haubenreich, P.N. and Engel, J.R. Nucl. Appl. Technol., 1970, 8, 118.

36 Sahin, S. and Ubeyli, M. Energy Conversion and Management, 2005, 46, 3185 (2005).

37 Robbins, G.D. and Braunstein, J. Molten salt reactor program, semi-annual progress report, ORNL-4449, 1969, 1970

38 Toth, L. M., Bates, J. B. and Boyd, G. E., J. Phys. Chem., 77(2), 216 (1973).

39 Heaton, R.J., Brookes, R.J., Madden, P.A., Salanne, M., Simon, C. Turq, P., J. Phys. Chem. 110, 11454 (2006).

40 Salanne, M., Simon, C. Turq, P., and Madden, P.A., J. Phys. Chem. B, 111, 4678-4684 (2007).

41 Loudon, R. Advances in Physics 50, 813 (2001).

42 Segall, M.D., Lindan, P.J.D., Probert, M.J., Pickard, C.J., Hasnip, P.J., Clark, S.J. and Payne, M.C., J. Phys. Condens. Matt. 14, 27172002.

43 Perdew, J.P. Phys. Rev. B 45, 132441992.

${ }^{44}$ Opium (http://opium.sourceforge.net/) Authors: Ramer, N.J. and Rappe, A.M.

45 Berghold, G., Mundy, C.J., Romero, A.H., Hütter, J., and Parrinello, M., Phys. Rev. B 61, 10040 (2000).

46 Buckingham, A.D., Adv. Chem. Phys., 12, 107 (1967).

47 Fowler. P.W. and Madden, P.A., Phys. Rev. B, 30, 6131 (1984).

48 Papatheodorou, G.N. and Dracopoulos, V.. Chem. Phys. Lett., 241, 345 (1995).

49 CERN Minuit Minimization package, authors James, F. and Roos, M., http://seal.web.cern.ch/seal/workpackages/mathlibs/minuit/home.html

50 Birnbaum, G. editor. Phenomena Induced by Intermolecular Interactions. Plenum, New York, (1985).

51 Dracopoulos, V. and Papatheodorou, G.N., Phys. Chem. Chem. Phys., 2, 2021 (2000).

52 Sharma, T. P., Kirby, R. D. and Jaswal, S. S.. Phys. Rev. B, 9(4), 1971 (1974).

53 Pavlatou, E.A., Madden, P.A. and Wilson, M., J. Chem. Phys. 107, 10446 (1997). 


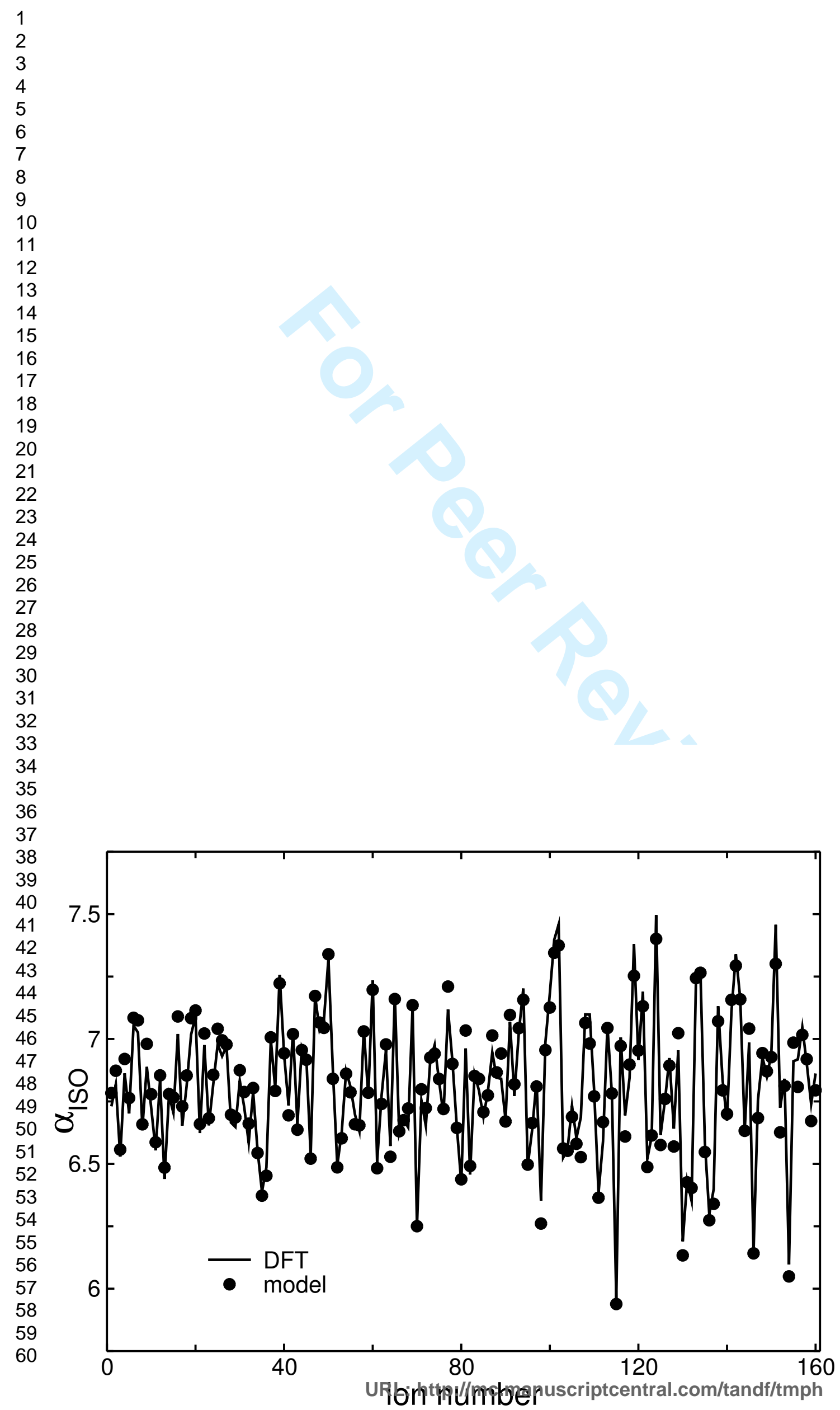




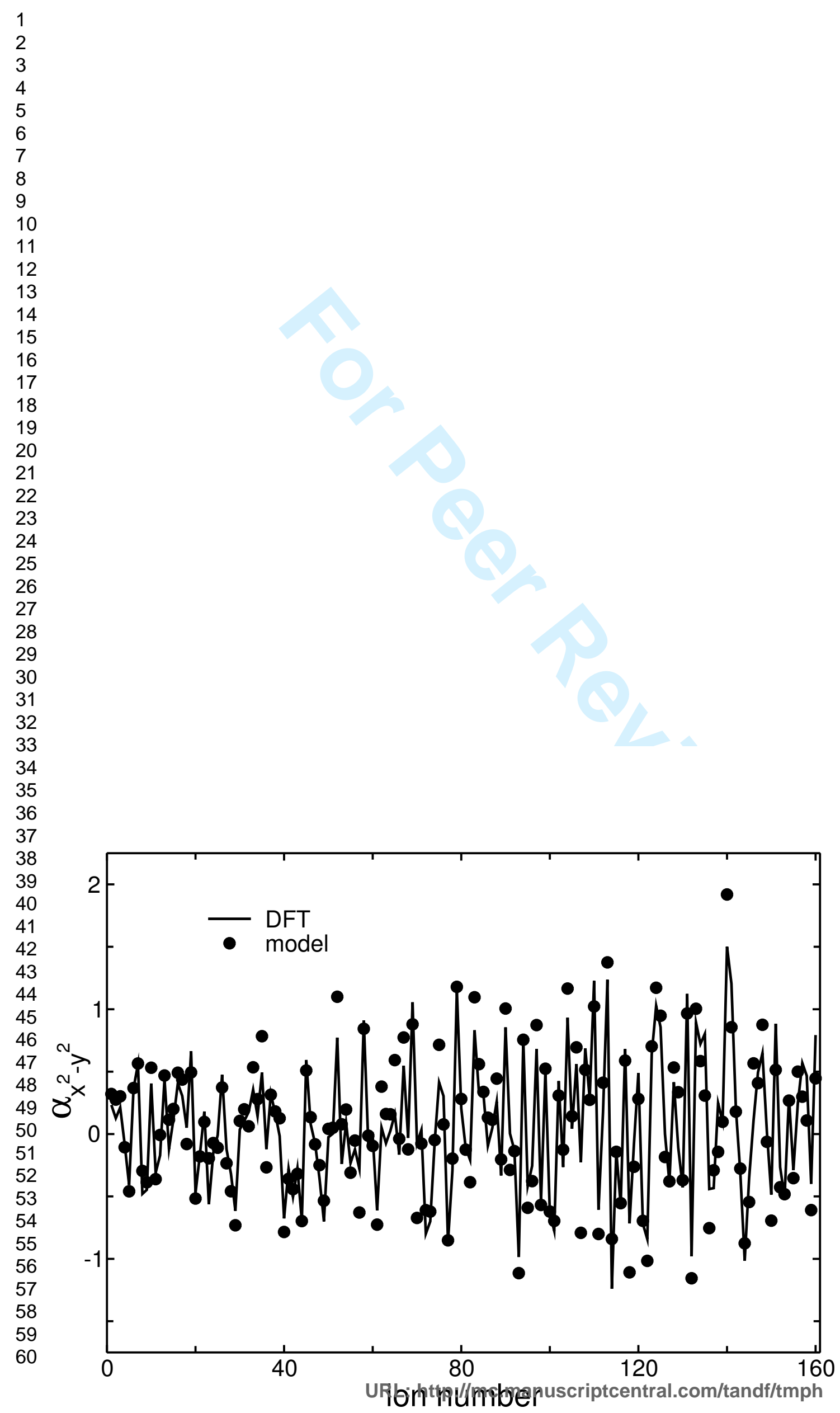




4

4
4

4
4

4
4
4

4

4

48

49

50

51
52

5

54

55

56

5

58

59

60

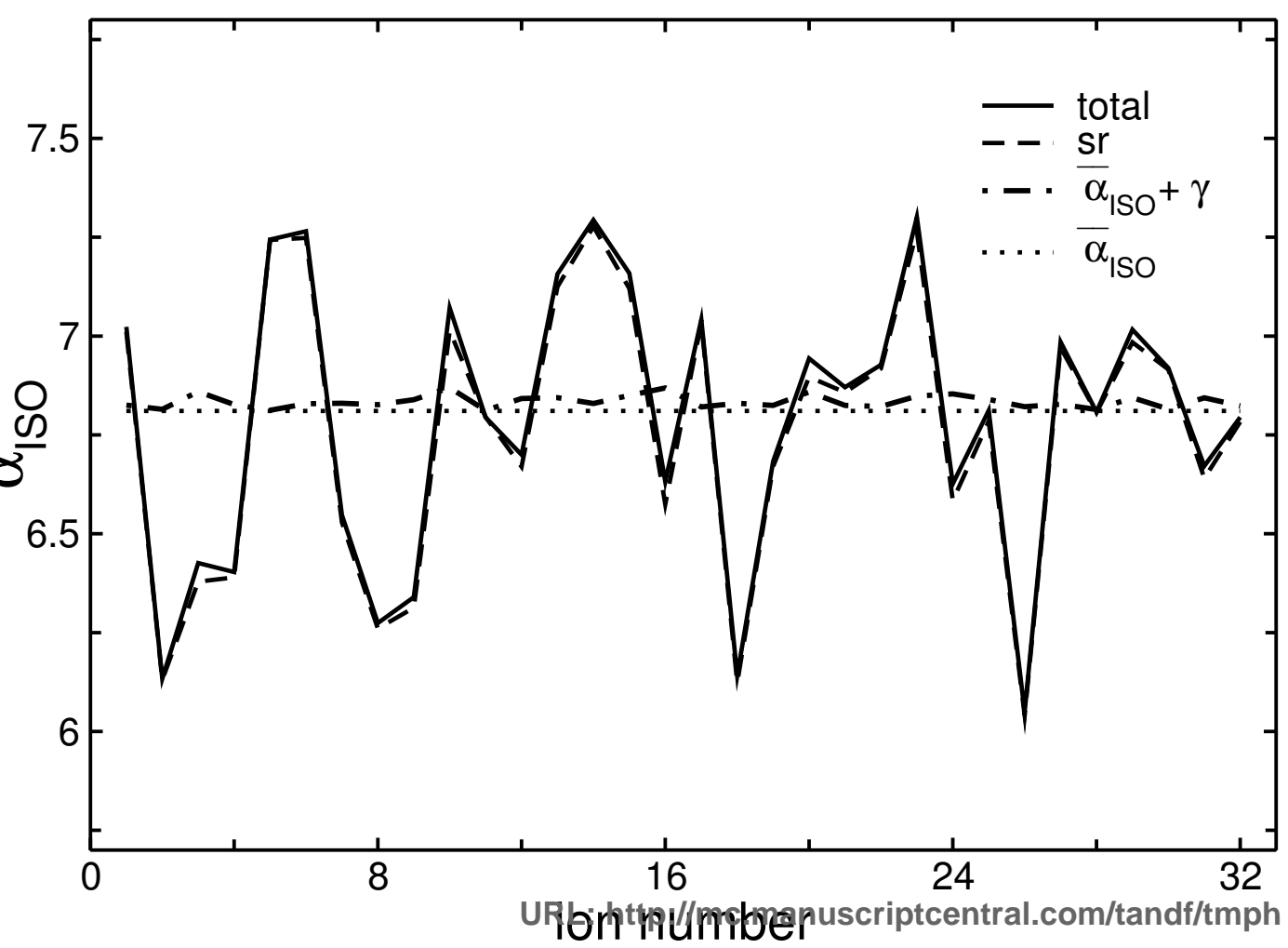




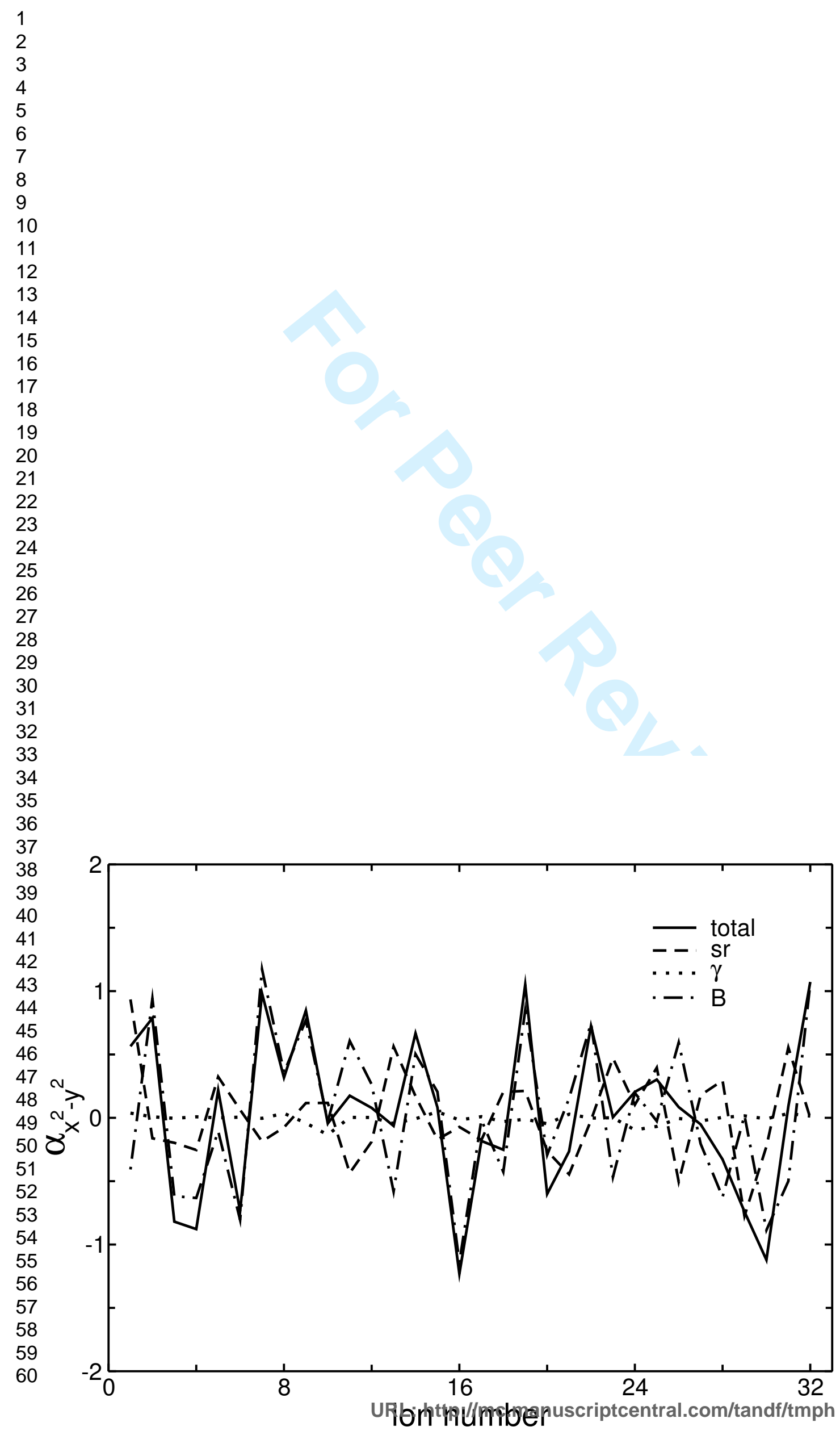




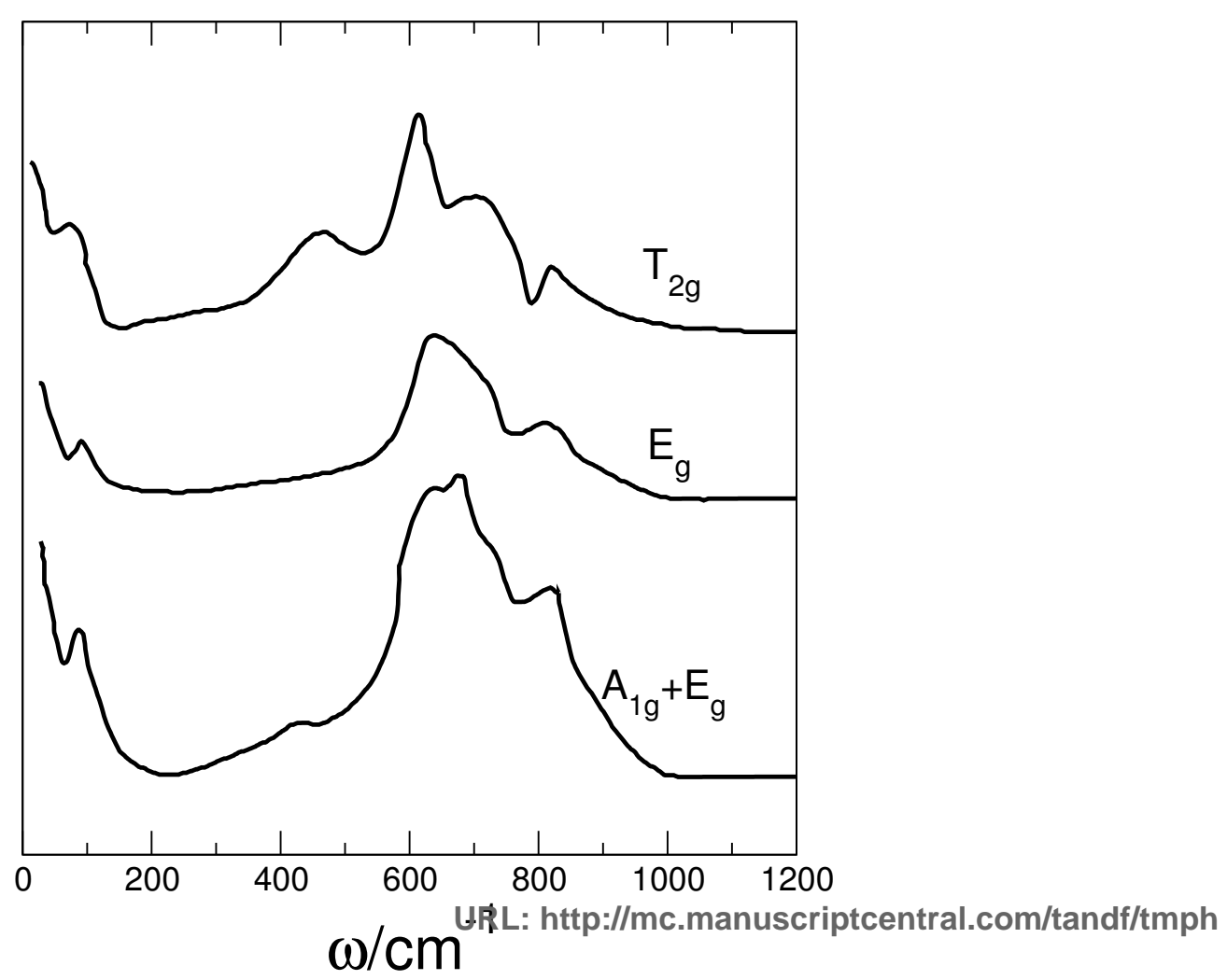




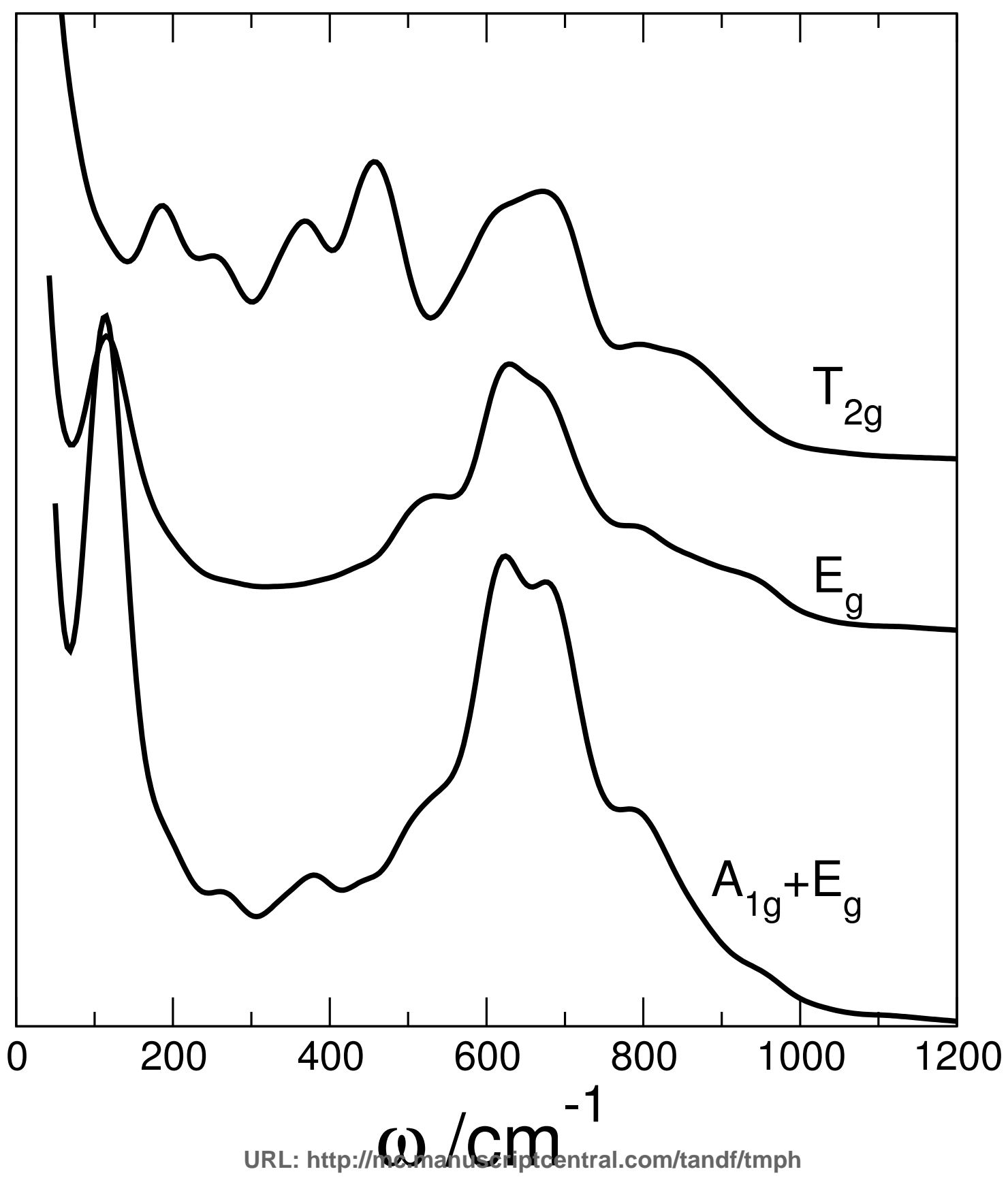




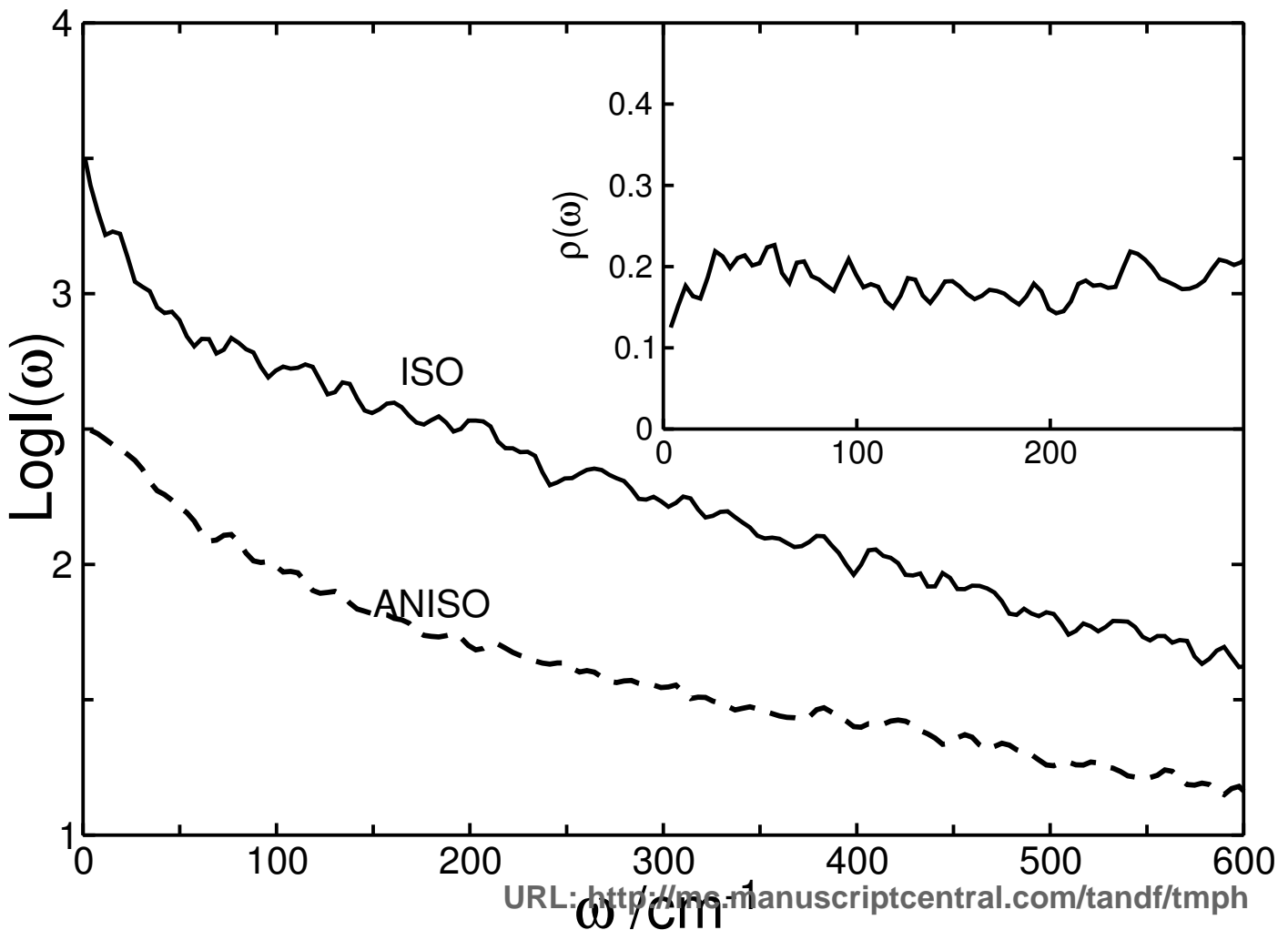




4
4

4

4
4

4
5

5

5

5

5

5

56

57

58

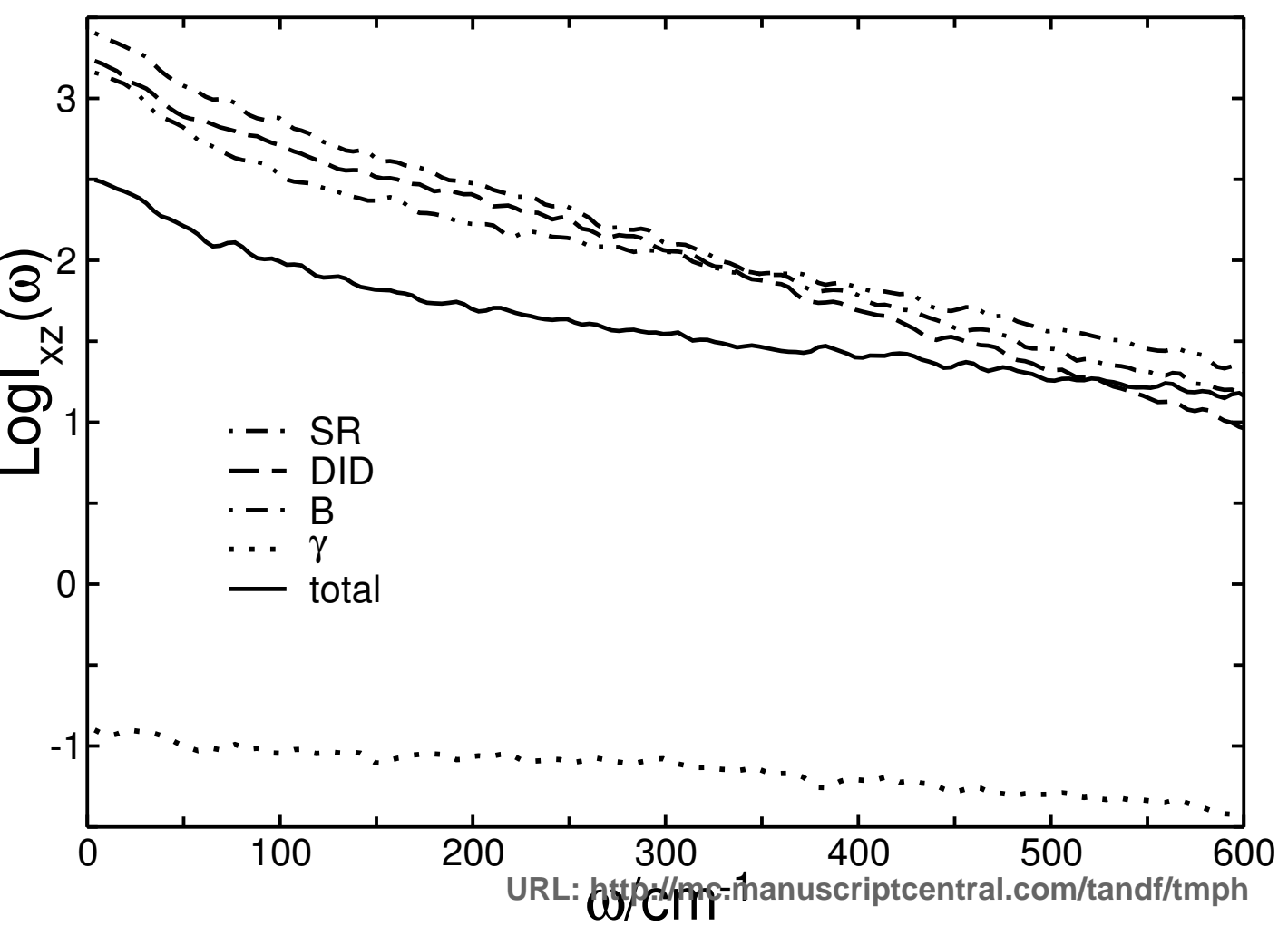




3

39

40

41

42

43

44

45

46

47

48

49

50

5

5

5

5

5

5

5

58

59

60

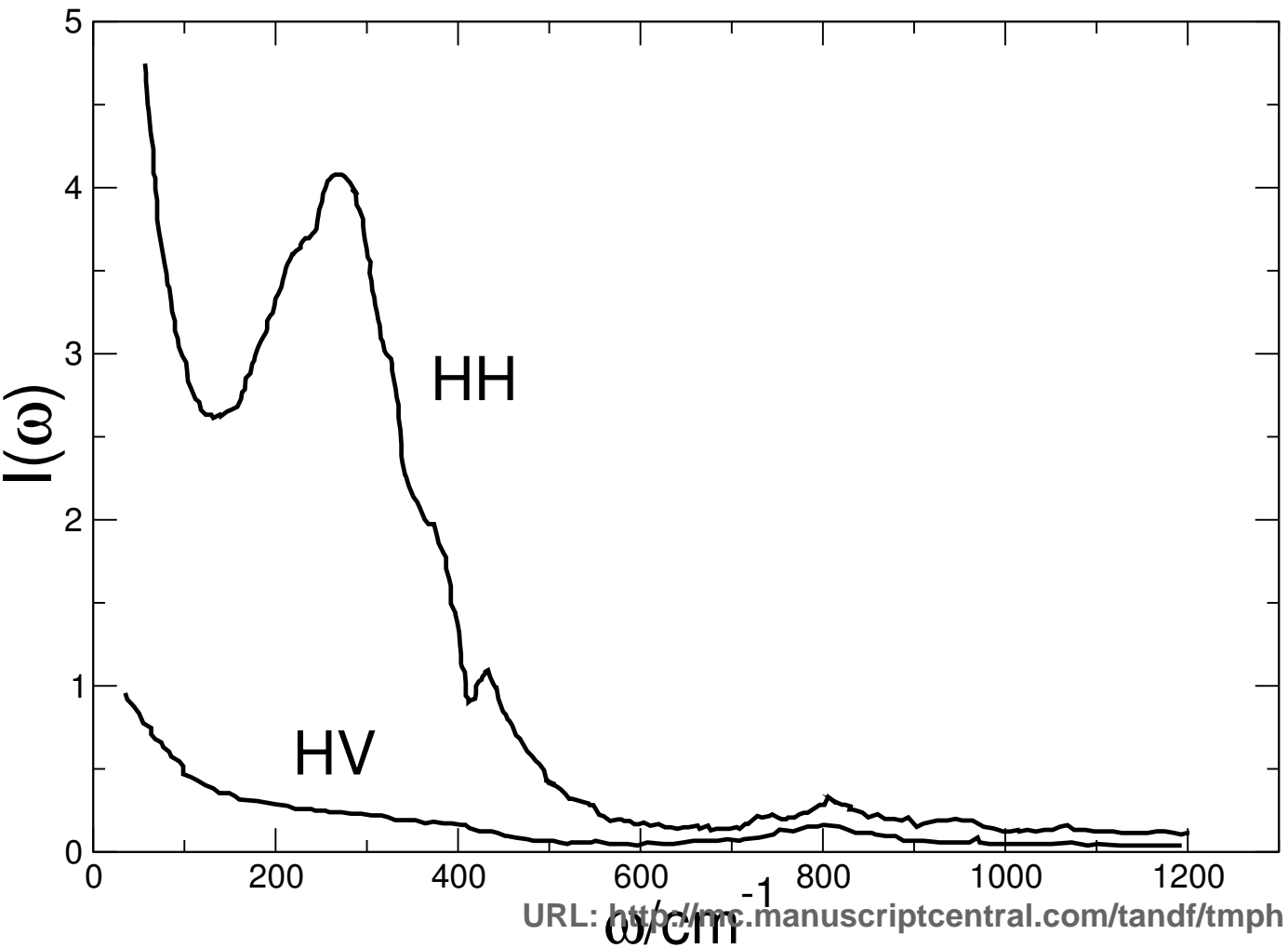




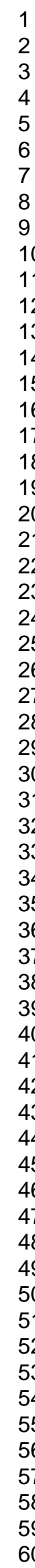

2

3

5

6

7

9

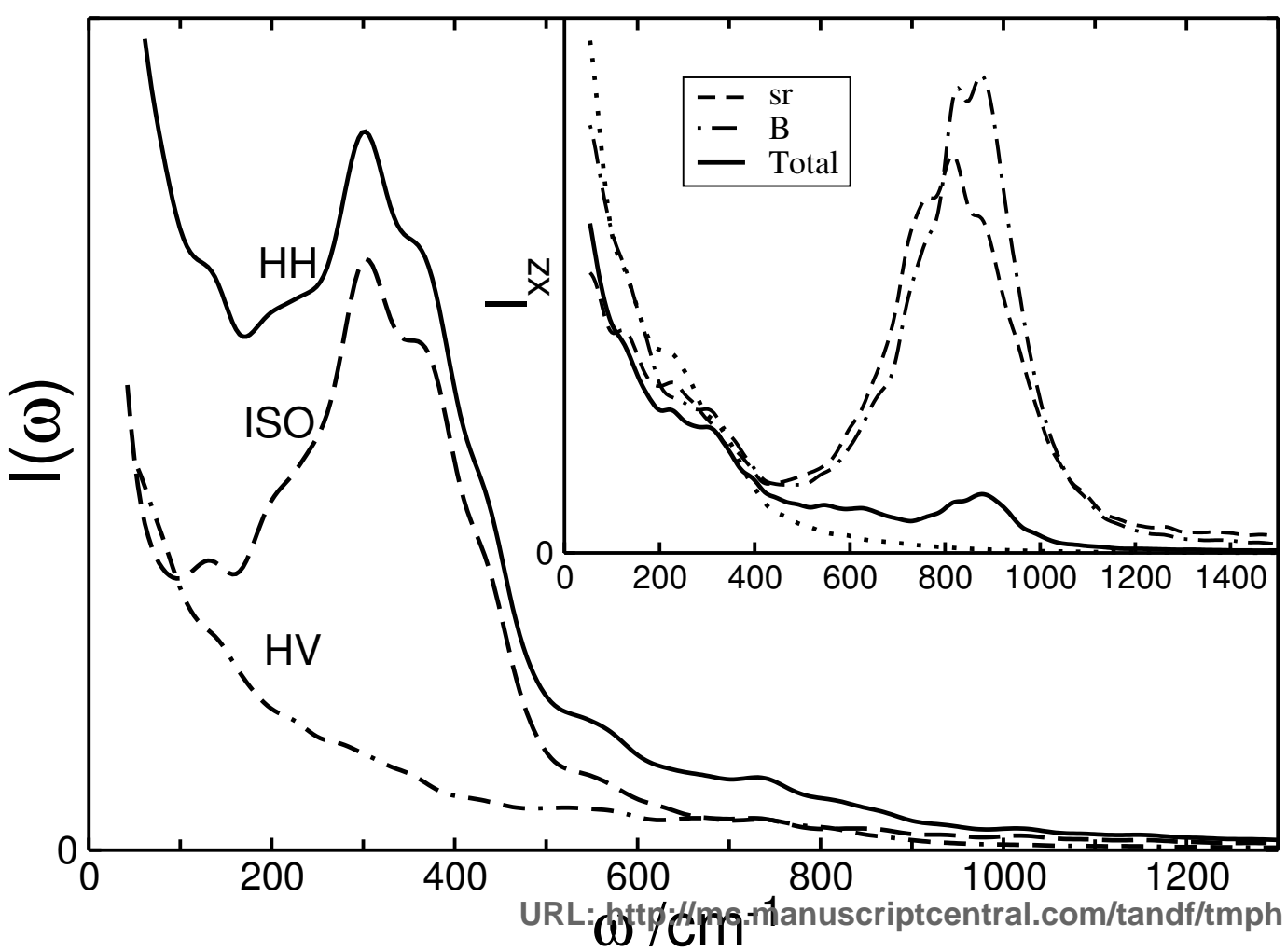


47

48

49

$50=$

51

52

53

54

55

56

57

58

59

60

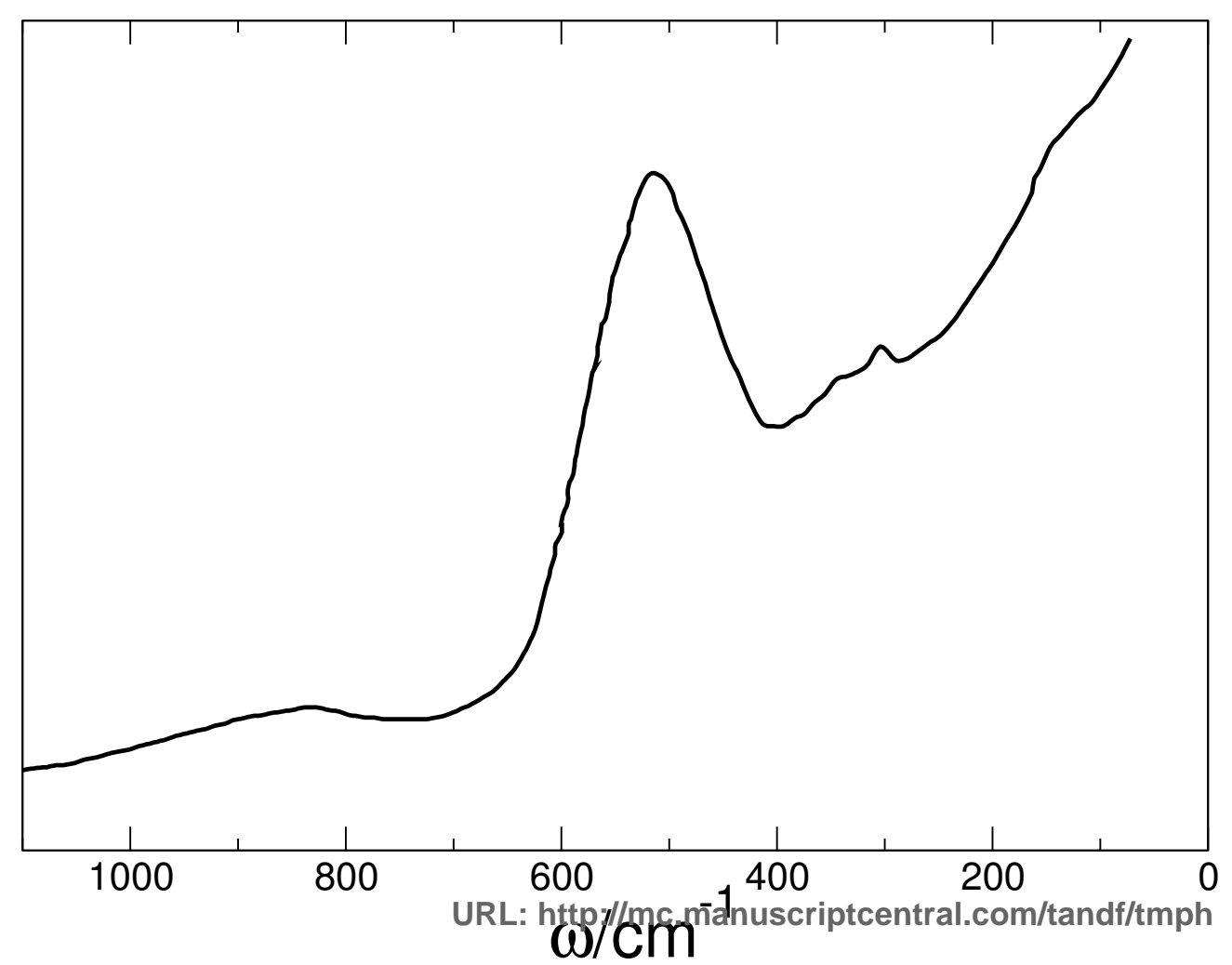




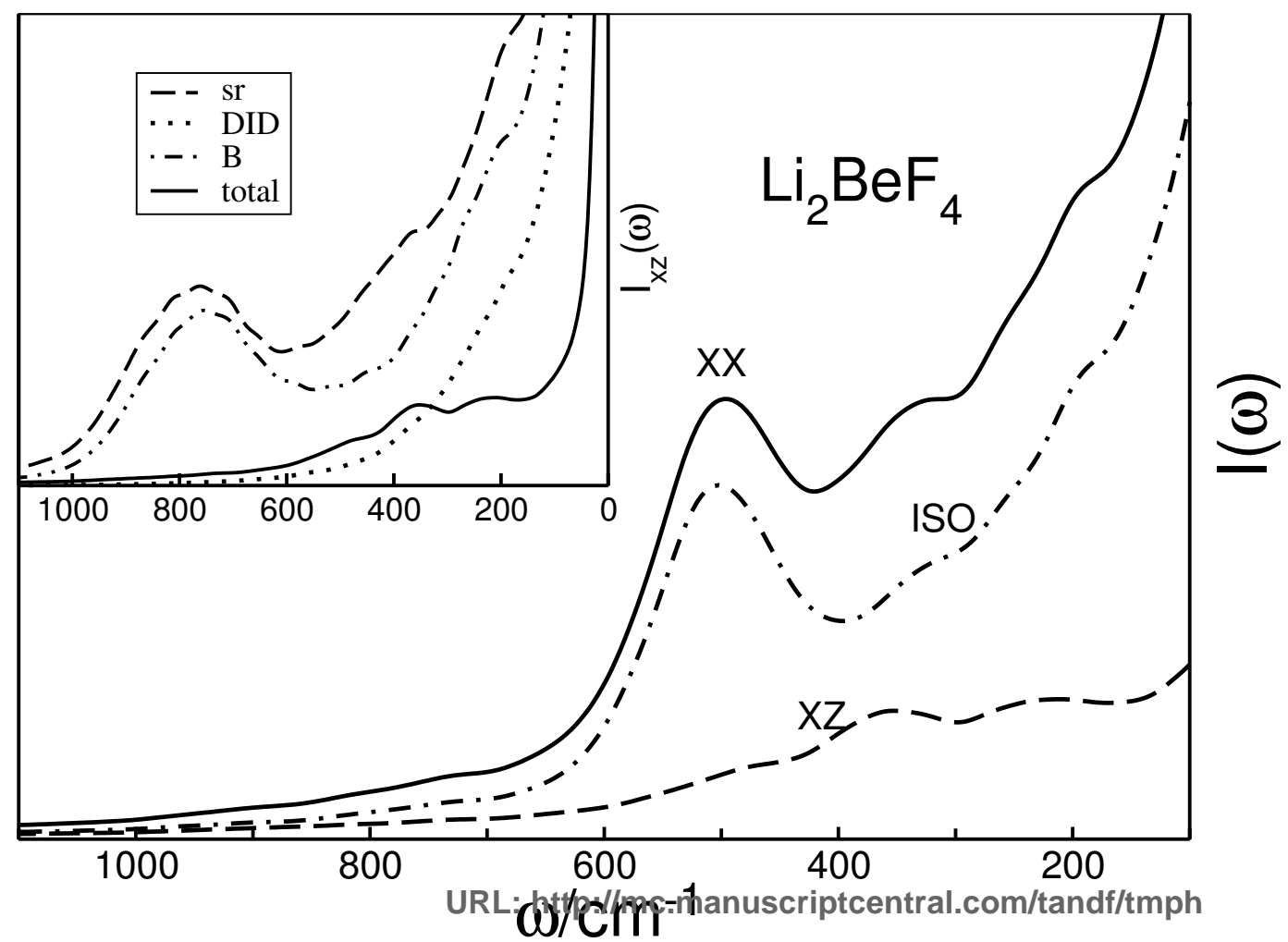

